\title{
Solving Variational Problems and Partial Differential Equations Mapping into General Target Manifolds
}

\author{
Facundo Mémoli * $\quad$ Guillermo Sapiro $^{\dagger} \quad$ Stanley Osher ${ }^{\ddagger}$
}

\begin{abstract}
A framework for solving variational problems and partial differential equations that define maps onto a given generic manifold is introduced in this paper. We discuss the framework for arbitrary target manifolds, while the domain manifold problem was addressed in [3]. The key idea is to implicitly represent the target manifold as the level-set of a higher dimensional function, and then implement the equations in the Cartesian coordinate system of this new embedding function. In the case of variational problem, we restrict the search of the minimizing map to the class of maps whose target is the levelset of interest. In the case of partial differential equations, we implicitly represent all the equation characteristics. We then obtain a set of equations that while defined on the whole Euclidean space, they are intrinsic to the implicit target manifold and map into it. This permits the use of classical numerical techniques in Cartesian grids, regardless of the geometry of the target manifold. The extension to open surfaces and submanifolds is addressed in this paper as well. In the latter case, the submanifold is defined as the intersection of two higher dimensional surfaces, and all the computations are restricted to this intersection. Examples of the applications of the framework here described include harmonic maps in liquid crystals, where the target manifold is an hypersphere; probability maps, where the target manifold is an hyperplane; chroma enhancement; texture mapping; and general geometric mapping between high dimensional surfaces.
\end{abstract}

\footnotetext{
* Instituto de Ingenieria Electrica, Universidad de la Republica, Montevideo, Uruguay.

${ }^{\dagger}$ Corresponding author: Electrical and Computer Engineering, University of Minnesota, Minneapolis, MN 55455, guille@ece.umn.edu

${ }^{\ddagger}$ UCLA Mathematics Department, Los Angeles, CA 90095.
} 


\section{Introduction}

In a number of applications in mathematical physics, image processing, computer graphics, and medical imaging, we have to solve variational problems and partial differential equations defined on a general manifold $\mathcal{M}$ (domain manifold), mapping the data onto another general manifold $\mathcal{N}$ (target manifold). That is, we deal with maps from $\mathcal{M}$ to $\mathcal{N}$. When these manifolds are for example three dimensional surfaces, the implementation of the corresponding gradient descent flow or the given PDE's is considerably elaborated. In [3] we have shown how to address this problem for general domain manifolds, while restricting the target manifolds $\mathcal{N}$ to the trivial cases of the Euclidean space or hyper-spheres. The key idea was to implicitly represent the domain surface as the (zero) level-set of a higher dimensional function $\phi$, and solve the PDE in the Cartesian coordinate system of this new embedding function. The technique was justified and demonstrated in [3]. It is the goal of this paper to show how to work with general target manifolds, and not just hyper-planes or hyper-spheres as previously reported in the literature. Inspired by [3], we also embed the target manifold $\mathcal{N}$ as the (zero) level-set of a higher dimensional function $\psi$. That is, when solving the gradient descent flow (or in general, the PDE), we guarantee that the map receives its values on the zero level-set of $\psi$. The map is defined on the whole space, although it never receives values outside of this level-set. Examples of applications of this framework include harmonic maps in liquid crystals $(\mathcal{N}$ is an hypersphere) and 3D surface warping [43]. In this last case, the basic idea is to find a smooth map between two given three dimensional surfaces. Due to the lack of the new frameworks introduced here and in [3], this problem is generally addressed in the literature after an intermediate mapping of the surfaces onto the plane is performed (see also [25, 46]). With these novel frameworks, direct three dimensional maps can be computed without any intermediate mapping, thereby eliminating their corresponding geometric distortions [31]. For this application, as in [43], boundary conditions are needed, and how to add them to the frameworks introduced here and in [3] is addressed in [31].

To introduce the ideas, in this paper we concentrate on flat domain manifolds. ${ }^{1}$ When combining this framework with the results on [3], we can of course work with general domains and then completely avoid other popular surface representations, like triangulated surfaces. We are then able to work with intrinsic equations, in Euclidean space and with classical numerics on Cartesian grids, regardless of the geometry of the involved domain and target manifolds. In addition to presenting the general theory, we also address the problem of target submanifolds and open surfaces. A number of theoretical results complement the algorithmic framework here described.

The implicit representation of surfaces here introduced for solving variational problems and PDE's is inspired in part by the level-set work of Osher and Sethian [33]. This work, and those that followed it, showed the importance of embedding deforming surfaces in higher dimensional functions, obtaining more robust and accurate numerical algorithms (and topological freedom). Note that in contrast with the level-set approach of Osher and Sethian, our target manifold is fixed, what is "deforming" is the dataset being mapped onto it.

Numerical schemes that solve gradient descent flows and PDE's onto generic target manifolds $\mathcal{N}$ (and spheres or surfaces in particular) will in general move the points outside of $\mathcal{N}$ due to numerical errors. The points will then need to be projected back, ${ }^{2}$ see for example [1,9] for the case of $\mathcal{N}$ being a sphere (where the projection is trivial, just a normalization). For general target manifolds, this projection means that for every point $p \in \mathbb{R}^{d}\left(\mathcal{N} \subset \mathbb{R}^{d}\right.$ ) we need to know the closest point to $p$ in $\mathcal{N}$. This means knowing the

\footnotetext{
${ }^{1}$ For completeness, we will present the general equations for both generic domain and target manifolds at the end of the paper. These equations are easily derived from [3] and the work presented in this paper.

${ }^{2}$ For particular flat target manifolds as the whole space $\mathbb{R}^{d}$ or as those in [34], the projection is not needed. Other authors, e.g., $[6,26]$, have avoided the projection step for particular cases, while in [48] the authors modify the given variational formulation to include the projection step.
} 
distance from every point $p \in \mathbb{R}^{d}$ to $\mathcal{N}$ (or at least all points in a band of $\mathcal{N}$ ). This is nothing else than an implicit representation of the target $\mathcal{N}$, being the particular embedding a distance function. This presents an additional justification for the framework here introduced.

In a number of applications, the surfaces are already given in implicit form, e.g., [5], therefore, the framework introduced in this paper it is not only simple and robust, but it is also natural in those applications. On the other hand, not all surfaces (manifolds) are originally represented in implicit form. When the target manifold $\mathcal{N}$ is simple, like hyper-spheres in the case of liquid crystals, the implicitation process is trivial. For generic surfaces, we need to apply an algorithm that transforms the given explicit representation into an implicit one. Although this is still a very active area of research, many very good algorithms have been developed, e.g., [14, 18, 27, 45].

\section{The Framework}

From now we assume that the target manifold $\mathcal{N}$ is given as the zero level set of a higher dimensional embedding $\psi: \mathbb{R}^{d} \rightarrow \mathbb{R}$, which we consider to be a distance function (this mainly simplifies the notation). For the case where $\mathcal{N}$ is a surface in three dimensional space for example, then $\psi: \mathbb{R}^{3} \rightarrow \mathbb{R}$. We also assume that the domain manifold $\mathcal{M}$ is flat and open (as mentioned in the introduction, general domain manifolds were addressed in [3]). We illustrate the basic ideas with a functional from the theory of harmonic maps. This is just a particular example (and a very important one), and from it will be clear how the same arguments can be applied to any given variational problem and PDE. In particular, it can be applied to common Navier-Stokes flows used in brain warping [31].

\subsection{Variational Formulation}

We search for necessary conditions for the functional $E[\vec{u}]$, defined by

$$
E[\vec{u}] \triangleq \int_{\mathcal{M}} e[\vec{u}] d_{\mathcal{M}} v
$$

where

$$
e[\vec{u}] \triangleq \frac{1}{2}\left\|\mathbf{J}_{\vec{u}}\right\|_{\mathcal{F}}^{2}
$$

to achieve a minimum. Here, $\|\cdot\|_{\mathcal{F}}^{2}=\sum_{i j}(\cdot)_{i j}^{2}$ is the norm of Frobenius and $\mathbf{J}_{\vec{u}}$ is the Jacobian of the map $\vec{u}: \mathcal{M} \rightarrow\{\psi=0\}$. Note that here we are already restricting the map to be onto the zero level-set of $\psi$, that is, onto the surface of interest $\mathcal{N}$ (the target manifold). This is what permits us to work with the embedding function and the whole space, while guaranteeing that the map will always be onto the target manifold, as desired. We use $\vec{\cdot}$ to note that for the most general case, the function is vectorial. Once again, this energy will be used throughout this paper to exemplify our framework. It will be clear after developing this example that the same arguments work for other variational formulations, as well as for generic PDE's defined onto generic surfaces.

Assume that $\vec{u}$ is a map minimizing $E(\cdot)$. Given $t>0$, we construct the variation

$$
\vec{v}_{t} \triangleq \vec{u}+t \vec{r}
$$

where $\vec{r}$ is a compact map $C^{\infty}$ in $\mathcal{M}$. For an arbitrary $x \in \mathcal{M}$, we will in general not obtain that $\vec{v}_{t}(x) \in$ $\{\psi=0\}$, that is, $\psi\left(\vec{v}_{t}(x)\right) \neq 0$. Therefore, this variation is not admissible. On the other hand, we can from it construct an admissible variation via 


$$
\vec{w}_{t} \triangleq \Pi_{\{\psi=0\}}\left(\vec{v}_{t}\right)
$$

where $\boldsymbol{\Pi}_{\{\psi=0\}}: R^{d} \rightarrow\{\psi=0\}$ is the projection operator onto $\{\psi=0\}$. Note that since $\psi$ is a signed distance function, we can simply write this projection operator onto $\{\psi=0\}$ as

$$
\Pi_{\{\psi=0\}}(\vec{\alpha})=\vec{\alpha}-\psi(\vec{\alpha}) \nabla \psi(\vec{\alpha}) .
$$

Let's now define

$$
\mathcal{E}(t) \triangleq E\left[\vec{w}_{t}\right]
$$

Since the energy achieves a minimum for $t=0$,

$$
\left.\dot{\mathcal{E}}_{0} \triangleq \frac{d E(t)}{d t}\right|_{(t=0)}=0 .
$$

Let's compute this first variation. We have that

$$
\dot{\mathcal{E}}_{0}=\left.\sum_{i j} \int_{\mathcal{M}}\left(\frac{\partial w_{t}^{i}}{\partial x_{j}} \frac{d\left(\frac{\partial w_{t}^{i}}{\partial x_{j}}\right)}{d t}\right)\right|_{t=0} d_{\mathcal{M}} v
$$

Moreover $\left(\mathbf{H}_{\psi}\right.$ stands for the Hessian of $\left.\psi\right)$,

$$
\begin{aligned}
\frac{\partial w_{t}}{\partial x_{j}} & =\left(\frac{\partial \vec{u}}{\partial x_{j}}+t \frac{\partial \vec{r}}{\partial x_{j}}\right)-\left(\nabla \psi\left(\vec{w}_{t}\right) \cdot\left(\frac{\partial \vec{u}}{\partial x_{j}}+t \frac{\partial \vec{r}}{\partial x_{j}}\right)\right) \nabla \psi\left(\vec{w}_{t}\right) \\
& -\psi\left(\vec{w}_{t}\right) \mathbf{H}_{\psi}\left(\vec{w}_{t}\right)\left(\frac{\partial \vec{u}}{\partial x_{j}}+t \frac{\partial \vec{r}}{\partial x_{j}}\right)
\end{aligned}
$$

and we observe that

$$
\left.\frac{\partial w_{t}}{\partial x_{j}}\right|_{(t=0)}=\frac{\partial \vec{u}}{\partial x_{j}}-\left(\nabla \psi(\vec{u}) \cdot \frac{\partial \vec{u}}{\partial x_{j}}\right) \nabla \psi(\vec{u})
$$

since $\psi(\vec{u})=0$. We can further simplify this observing that $0=\frac{\partial \psi(\vec{u})}{\partial x_{j}}=\nabla \psi(\vec{u}) \cdot \frac{\partial \vec{u}}{\partial x_{j}}$. Therefore,

$$
\left.\frac{\partial w_{t}}{\partial x_{j}}\right|_{(t=0)}=\frac{\partial \vec{u}}{\partial x_{j}}
$$

With a bit of further simple analysis we can compute the additional derivative, $\frac{d\left(\frac{\partial w_{t}^{i}}{\partial x_{j}}\right)}{d t}=\frac{\partial\left(\frac{d w_{t}^{i}}{d t}\right)}{\partial x_{j}}$. This change in the order of derivatives is done in order to immediately evaluate the result at $t=0$, thereby simplifying the following derivative. Following in an similar form, we obtain

$$
\frac{d w_{t}^{i}}{d t}=\vec{r}-\left(\nabla \psi\left(\vec{w}_{t}\right) \cdot \vec{r}\right) \nabla \psi\left(\vec{w}_{t}\right)-\psi\left(\vec{w}_{t}\right) \mathbf{H}_{\psi}\left(\vec{w}_{t}\right) \vec{r}
$$

and

$$
\left.\frac{d w_{t}^{i}}{d t}\right|_{(t=0)}=\vec{r}-(\nabla \psi(\vec{u}) \cdot \vec{r}) \nabla \psi(\vec{u}) .
$$


Combining the above computations all together we obtain

$$
\begin{aligned}
\left.\frac{d\left(\frac{\partial w_{t}^{i}}{\partial x_{j}}\right)}{d t}\right|_{(t=0)} & =\frac{\partial\left(\left.\frac{d w_{t}^{i}}{d t}\right|_{(t=0)}\right)}{\partial x_{j}} \\
& =\frac{\partial \vec{r}}{\partial x_{j}}-\nabla \psi(\vec{u})\left\{\frac{\partial \vec{r}}{\partial x_{j}} \cdot \nabla \psi(\vec{u})+\mathbf{H}_{\psi}\left(\vec{r}, \frac{\partial \vec{u}}{\partial x_{j}}\right)\right\}-(\vec{r} \cdot \nabla \psi(\vec{u}))\left(\mathbf{H}_{\psi} \frac{\partial \vec{u}}{\partial x_{j}}\right) .
\end{aligned}
$$

Following from (3) we have that ${ }^{3}$

$$
\begin{aligned}
\dot{\mathcal{E}}_{0} & =\left.\sum_{j} \int_{\mathcal{M}}\left(\frac{\partial \vec{w}_{t}}{\partial x_{j}} \frac{d\left(\frac{\partial \vec{w}_{t}}{\partial x_{j}}\right)}{d t}\right)\right|_{t=0} d_{\mathcal{M}} v \\
& =\sum_{j} \int_{\mathcal{M}}\left\{\frac{\partial \vec{r}}{\partial x_{j}} \cdot \frac{\partial \vec{u}}{\partial x_{j}}-(\vec{r} \cdot \nabla \psi(\vec{u})) \mathbf{H}_{\psi}\left[\frac{\partial \vec{u}}{\partial x_{j}}, \frac{\partial \vec{u}}{\partial x_{j}}\right]\right\} d_{\mathcal{M}} v .
\end{aligned}
$$

Now, applying the divergence theorem we conclude the computation. We first write

$$
\sum_{i j} \int_{\mathcal{M}} \frac{\partial \vec{r}}{\partial x_{j}} \cdot \frac{\partial \vec{u}}{\partial x_{j}} d_{\mathcal{M}} v=\sum_{i} \int_{\mathcal{M}} \nabla r^{i} \cdot \nabla u^{i} d_{\mathcal{M}} v
$$

and then apply the fact $\nabla r^{i} \cdot \nabla u^{i}=\nabla \cdot\left(r^{i} \nabla u^{i}\right)-r^{i} \Delta u^{i}$, together with the divergence theorem, to obtain (n stands for the outward unit normal to $\partial \mathcal{M}$ ).

$$
\sum_{i j} \int_{\mathcal{M}} \frac{\partial \vec{r}}{\partial x_{j}} \cdot \frac{\partial \vec{u}}{\partial x_{j}} d_{\mathcal{M}} v=\sum_{i} \int_{\partial \mathcal{M}} r^{i} \frac{\partial u^{i}}{\partial \mathbf{n}} d S-\int_{\mathcal{M}} r^{i} \Delta u^{i} d_{\mathcal{M}} v
$$

To conclude we put together this last expression with (8), and after some algebra we obtain that $\dot{\mathcal{E}}_{0}$ is equal to

$$
\int_{\partial \mathcal{M}} \vec{r} \cdot \mathbf{J}_{\vec{u}} \mathbf{n} d S-\int_{\mathcal{M}} \vec{r} \cdot\left\{\Delta \vec{u}+\left(\sum_{k} \mathbf{H}_{\psi}\left[\frac{\partial \vec{u}}{\partial x_{k}}, \frac{\partial \vec{u}}{\partial x_{k}}\right]\right) \nabla \psi(\vec{u})\right\} d_{\mathcal{M}} v
$$

The boundary condition is eliminated since the support of $\vec{r}$ is compactly included in $\mathcal{M}$. To eliminate the additional term for an arbitrary $\vec{r}$ we must impose

$$
\Delta \vec{u}+\left(\sum_{k} \mathbf{H}_{\psi}\left[\frac{\partial \vec{u}}{\partial x_{k}}, \frac{\partial \vec{u}}{\partial x_{k}}\right]\right) \nabla \psi(\vec{u})=0 .
$$

This gives the corresponding Euler-Lagrange for the given variational problem. Note once again from our computations that in spite that all the terms "live" in the Euclidean space embedding the target manifold, $\vec{u}$ will always map onto the level-set of interest, $\{\psi=0\}$, and therefore, onto the surface of interest. This is guaranteed by this equation, no additional computations are needed. This is the beauty of the approach, while working freely on the Euclidean space (and therefore with Cartesian numerics), we can guarantee that the equations are intrinsic to the given surfaces of interest. We will further verify this in $\S 2.4$ to help the reader with the intuition behind this framework.

\footnotetext{
${ }^{3}$ We have used as before the notation $A[\vec{x}, \vec{y}]=\vec{y}^{T} A \vec{x}$
} 


\subsection{Harmonic Maps}

The expressions derived in the previous sections come from the theory of harmonic maps, e.g., [4, 7, 11 , $13,15,16,20,23,35,38,39,40]$. In general, harmonic maps are defined as maps between two manifolds $(\mathcal{M}, g)$ and $(\mathcal{N}, h)$ minimizing the energy

$$
E[\vec{u}] \triangleq \int_{\mathcal{M}} e[\vec{u}] d V_{\mathcal{M}}
$$

where in local coordinates the energy density $e[\vec{u}]$ is given by

$$
e[\vec{u}](x) \triangleq \frac{1}{2} g^{p q}(x) h_{i j}(\vec{u}(x)) \frac{\partial u^{i}}{\partial x_{p}} \frac{\partial u^{j}}{\partial x_{q}} .
$$

We have used Einstein's summation here, where repeated indices indicate summation with respect to this index, together with the usual notation for tensors. ${ }^{4}$ When both the domain and target manifolds are represented explicitly, the classical case, the Euler-Lagrange equation corresponding to this energy is given by (see [38])

$$
\Delta_{\mathcal{M}} u^{l}+\Gamma_{i j}^{l}(\vec{u}) g^{\alpha \beta} \frac{\partial u^{i}}{\partial x^{\alpha}} \frac{\partial u^{j}}{\partial x^{\beta}}=0
$$

where $\Delta_{\mathcal{M}}$ is the Laplace-Beltrami operator (reduced to the regular Laplacian for the case of flat domain manifolds) and $\Gamma_{i j}^{l}(\vec{u})$ stands for the Christoffel symbols of the target manifold evaluated at $\vec{u}$. Note that the first component, the Laplace-Beltrami, addresses the domain manifold, while the second term addresses the target manifold. By embedding the target manifold, we are changing the Christoffel symbols (expressing them in implicit form, see below), ${ }^{5}$ while the work in [3] changed the other terms, since the embedding was done to the domain manifold, see $\S 5$.

As an example, let's see what happens with the above energy for the Euclidean case. Since both metrics are proportional to the identity,

$$
e[\vec{u}](x)=\frac{K}{2} \sum_{i j}\left(\frac{\partial u^{i}}{\partial x_{j}}\right)^{2}
$$

which is just a constant multiplying $\left\|\mathbf{J}_{\vec{u}}\right\|_{\mathcal{F}}^{2}$. Therefore, the energy defined in the previous case is just a particular case of harmonic maps. In general, this energy can be used in problems such as color image denoising and directions denoising [40,41], as a regularization term for ill-possed problems defined on general surfaces [17], for general denoising [37, 44], for models of liquid crystals, and as a component of a system for surface mapping and matching $[13,31,46]$.

\subsubsection{An(other) Informal Calculation}

We now present an additional computation that connects in a deep way the implicit framework with harmonic maps. We consider the harmonic energy density given in (14) for the planar domain manifold case $\left(g_{i j}=\right.$ $\left.\delta_{i j}\right)$. We can simplify things to obtain

$$
e[\vec{u}](x)=\frac{1}{2} h_{i j}(\vec{u}(x)) \frac{\partial u^{i}}{\partial x_{p}} \frac{\partial u^{j}}{\partial x_{p}}=\frac{1}{2} \sum_{p} \mathbf{h}\left[\vec{u}_{x_{p}}, \vec{u}_{x_{p}}\right]
$$

\footnotetext{
${ }^{4}\left(g^{-1}\right)_{i j} \triangleq g^{i j}$.

${ }^{5}$ Or alternatively, the second fundamental form of the target manifold.
} 
We know that $\mathbf{\Pi}_{\nabla \psi}=\mathbf{I}-\nabla \psi \nabla \psi^{T}$ can be thought of as the inverse of the target manifold's metric tensor. But since $\nabla \psi$ is a zero eigenvalue eigenvector for $\Pi_{\nabla \psi}$, it will be a $\infty$ eigenvalue eigenvector for $\boldsymbol{\Pi}_{\nabla \psi}^{-1}$. Then, we can't use the identification $\mathbf{h}=\left(h_{i j}\right) \leftrightarrow \mathbf{\Pi}_{\nabla \psi}^{-1}$ in the above expression for the energy density. However, we can proceed as follows. Take $\epsilon>1$ and define the metric ${ }^{6}$

$$
\mathbf{h}^{\epsilon} \triangleq\left(\epsilon \mathbf{I}-\nabla \psi \nabla \psi^{T}\right)^{-1}
$$

one can then compute the inverse as (it's an elementary formula, see for example [24])

$$
\mathbf{h}^{\epsilon}=\frac{1}{\epsilon}\left(\mathbf{I}+\frac{\nabla \psi \nabla \psi^{T}}{\epsilon-1}\right)
$$

The energy density can be rewritten as (we will use a subindex $\epsilon$ )

$$
e_{\epsilon}[\vec{u}](x)=\frac{1}{2 \epsilon}\left(\sum_{i}\left\|\vec{u}_{x_{i}}\right\|^{2}+\frac{1}{\epsilon-1} \sum_{i}\left|\vec{u}_{x_{i}} \cdot \nabla \psi\right|^{2}\right)
$$

After computing the variational derivative for the functional $\int_{\mathcal{M}} e_{\epsilon}[\vec{u}](x) d x$ we obtain that $\vec{u}$ must satisfy

$$
\Delta \vec{u}+\frac{1}{\epsilon-1}\left(\sum_{i} \mathbf{H}_{\psi}\left[\vec{u}_{x_{i}}, \vec{u}_{x_{i}}\right]+\Delta \vec{u} \cdot \nabla \psi\right) \nabla \psi=0
$$

By multiplying all the terms in the above equation by $\epsilon-1$ and letting $\epsilon \rightarrow 1$ we find that the expression between brackets must vanish. As we will see in $\S 2.4$, what's between brackets is nothing but $\Delta \nu(x)$ where $\nu(x)=\psi(\vec{u}(x))$. So $\nu$ is a harmonic function in $\mathcal{M}$. It is also evident that $\nu$ satisfies Dirichlet boundary conditions if $\vec{u}$ does, and since we are trying to map things from $\mathcal{M}$ to $\mathcal{N}$, those boundary conditions for $\vec{u}$ must be such that $\operatorname{dist}(\vec{u}(x), \mathcal{N})=0$ for $x \in \mathcal{M}$, so $\left.\nu\right|_{\partial \mathcal{M}}=0$. Then we conclude that $\nu$ must be zero everywhere in $\mathcal{M}$.

\subsection{The Mapping Flow}

The PDE used for solving the harmonic energy is given by its corresponding gradient descent flow. This gradient descent is given by

$$
\frac{\partial u^{i}}{\partial t}=\Delta u^{i}+\sum_{k=1}^{d} \mathbf{H}_{\psi}(\vec{u})\left[\frac{\partial \vec{u}}{\partial x_{k}}, \frac{\partial \vec{u}}{\partial x_{k}}\right] \frac{\partial \psi}{\partial u^{i}}(\vec{u})
$$

where the initial datum $\vec{u}_{0}$ is given by the vector field we want to process, together with Neumann boundary conditions:

$$
\left\{\begin{array}{l}
\vec{u}(x, 0)=\vec{u}_{0}(x), \quad x \in \mathcal{M} \\
\left.\mathbf{J}_{\vec{u}} \mathbf{n}\right|_{\partial \mathcal{M}}=\mathbf{0} .
\end{array}\right.
$$

The use of Neumann boundary conditions needs to be justified. In the scalar case, one has the evolution problem

$$
\left\{\begin{array}{l}
I_{t}(x, t)=\Delta I(x, t) \quad x \in \mathcal{M}, t \geq 0 \\
I(x, 0)=I_{0}(x), \quad x \in \mathcal{M} \\
\left.\nabla I \cdot \mathbf{n}\right|_{\partial \mathcal{M}}=\mathbf{0}
\end{array}\right.
$$

\footnotetext{
${ }^{6}$ Since $\epsilon>1$ all the eigenvalues are positive.
} 
We observe that the quantity $\sigma(t) \triangleq \int_{\mathcal{M}} I(x, t) d_{\mathcal{M}} v$ remains constant,

$$
\dot{\sigma}(t)=\int_{\mathcal{M}} I_{t}(x, t) d_{\mathcal{M}} v=\int_{\mathcal{M}} \Delta I(x, t) d_{\mathcal{M}} v=\int_{\mathcal{M}} \nabla \cdot(\nabla I) d_{\mathcal{M}} v=\int_{\partial \mathcal{M}} \nabla I \cdot \mathbf{n} d_{\mathcal{M}} s=0
$$

thereby imposing the boundary conditions.

One wonders which quantity is preserved thru time by the flow in the general case, when imposing the boundary condition (17). We illustrate this for the particular case of $\mathcal{N}=S^{1}$. In this case, the evolution equations are given by (see also $\S 2.4$ below)

$$
\left\{\begin{array}{l}
X_{t}=\Delta X+\left(\|\nabla X\|^{2}+\|\nabla Y\|^{2}\right) X \\
Y_{t}=\Delta Y+\left(\|\nabla X\|^{2}+\|\nabla Y\|^{2}\right) Y
\end{array}\right.
$$

The Neumann boundary conditions for this case are written as

$$
\nabla X \cdot \mathbf{n}=\nabla Y \cdot \mathbf{n}=0 \text { in } \partial \mathcal{M}
$$

Transforming to polar coordinates $(\rho, \theta)$ one finds that the evolution equations (for smooth initial data, and at least for some time) are (see also [35])

$$
\left\{\begin{array}{l}
\theta_{t}=\Delta \theta \\
\rho_{t}=0
\end{array}\right.
$$

with boundary conditions

$$
\nabla \theta \cdot \mathbf{n}=0 \text { in } \partial \mathcal{M}
$$

Again one finds that $\int_{\mathcal{M}} \theta(x, t) d_{\mathcal{M}} v$ is constant.

In the most general case, when the target manifold is arbitrary, one might guess that the intrinsic barycenter ${ }^{7}$ of the map is preserved through time, since that's exactly what the particular cases given above show us. However, to the best of our knowledge, there is not such a result in the literature of harmonic maps, and the conservation of the barycenter is only obtained when constraints are added. The examples discussed above still motivate the use of Neumann boundary conditions.

\subsection{Simple Verifications}

We now illustrate that the Euler-Lagrange (12), and its corresponding gradient descent flow (16), are the extension for implicit targets of common equations derived in the literature for explicitly represented manifolds. We also explicitly show that the flow equation guarantees, as expected from the derivation above, that if the initial datum is on the target manifold, it will remain on it. We also express the second fundamental form of a manifold that is implicitly represented. All these results will help to further illustrate the approach and verify its correctness.

\footnotetext{
${ }^{7}$ The intrinsic barycenter $G$ of the map $\vec{u}: \Omega \rightarrow \mathcal{N}$ is defined by $G=\operatorname{argmin}_{p \in \mathcal{N}} \frac{1}{2} \int_{\Omega} d_{\mathcal{N}}^{2}(p, \vec{u}(x)) d x$. See [10] for more details on the barycenter.
} 


\section{Geodesics as Harmonic Maps}

It is well known, see $[15,16,36]$, that arc-length parameterized geodesics on the manifold $\mathcal{N}$ satisfy the harmonic maps PDE. If we assume isotropic and homogeneous metric over $\mathcal{N}$, we end up having that (arclength parameterized) geodesics must satisfy

$$
\ddot{\gamma}+\mathbf{H}_{\psi}[\dot{\gamma}, \dot{\gamma}] \nabla \psi(\gamma)=0
$$

\section{Liquid Crystals}

One of the most popular examples of harmonic maps is given when the target manifold $\mathcal{N}$ is an hypersphere. That is, the map is onto $S^{d-1}$. In this case, the embedding (signed distance) function is simply $\psi(\vec{y})=\|\vec{y}\|-1, \vec{y} \in \mathbb{R}^{d}$. From this, $\nabla \psi(\vec{y})=\frac{\vec{y}}{\|\vec{y}\|}$ and $\left(\mathbf{H}_{\psi}(\vec{y})\right)_{i j}=\frac{\delta_{i j}}{\|\vec{y}\|}-\frac{y_{i} y_{j}}{\|\vec{y}\|^{3}}$. We also have that $\mathbf{H}_{\psi}(\vec{u}(x))\left[\frac{\partial \vec{u}}{\partial x_{k}}, \frac{\partial \vec{u}}{\partial x_{k}}\right]=\delta_{i j} \frac{\partial u^{i}}{\partial x_{k}} \frac{\partial u^{j}}{\partial x_{k}}-\frac{\partial u^{i}}{\partial x_{k}} \frac{\partial u^{j}}{\partial x_{k}} u_{i} u_{j}$, since $\|\vec{u}\|=1$. In addition, $u^{i} \frac{\partial u^{i}}{\partial x_{k}}=0$, fact simply obtained taking derivatives with respect to $x_{k}$. We then obtain that $\frac{\partial u^{i}}{\partial x_{k}} \frac{\partial u^{j}}{\partial x_{k}} u_{i} u_{j}=\left(\frac{\partial u^{i}}{\partial x_{k}} u_{i}\right)^{2}=0$, and $\sum_{k=1}^{d} \mathbf{H}_{\psi}(\vec{u}(x))\left[\frac{\partial \vec{u}}{\partial x_{k}}, \frac{\partial \vec{u}}{\partial x_{k}}\right]=\sum_{i k}\left(\frac{\partial u^{i}}{\partial x_{k}}\right)^{2}=\left\|\mathbf{J}_{\vec{u}}(x)\right\|_{\mathcal{F}}^{2}$. Therefore, the corresponding diffusion equation from (16) is

$$
\frac{\partial \vec{u}}{\partial t}=\Delta \vec{u}+\left\|\mathbf{J}_{\vec{u}}\right\|_{\mathcal{F}}^{2} \vec{u}
$$

which is exactly the well known gradient descent flow for this case.

\section{Mapping Restriction onto the Zero Level-Set}

We now explicitly show that if the initial datum belongs to the target surface given by the zero level-set of $\psi$, then the solution to the diffusion flow (16) also belongs to this level-set. This further shows the correctness of our approach.

We basically need to show that $\psi(\vec{u}(x, t))=0, \forall x \in \mathcal{M}, \forall t \geq 0$. If the initial datum is on $\{\psi=0\}$, then this property is true for $t=0$. Let's define $\nu(x, t)=\psi(\vec{u}(x, t))$. Then ${ }^{8}$

$$
\frac{\partial \nu}{\partial t}=\nabla \psi(\vec{u}) \cdot \frac{\partial \vec{u}}{\partial t}=\Delta \vec{u} \cdot \nabla \psi(\vec{u})+\sum_{k=1}^{d} \mathbf{H}_{\psi}(\vec{u})\left[\frac{\partial \vec{u}}{\partial x_{k}}, \frac{\partial \vec{u}}{\partial x_{k}}\right]
$$

since $\psi$ is a distance function. In addition, $\frac{\partial \nu}{\partial x_{i}}=\nabla \psi(\vec{u}) \cdot \frac{\partial \vec{u}}{\partial x_{i}}$, and then

$$
\frac{\partial^{2} \nu}{\partial x_{i}^{2}}=\left(\mathbf{H}_{\psi}(\vec{u}) \frac{\partial \vec{u}}{\partial x_{i}}\right) \cdot \frac{\partial \vec{u}}{\partial x_{i}}+\nabla \psi(\vec{u}) \cdot \frac{\partial^{2} \vec{u}}{\partial x_{i}^{2}} .
$$

Adding on $i=1, \ldots, d$, it follows that $\frac{\partial \nu}{\partial t}=\Delta \nu$, meaning that $\nu$ verifies the heat flow. In addition to this, $\left.\frac{\partial \nu}{\partial \mathbf{n}}\right|_{\partial \mathcal{M}}=\nabla_{x}(\psi(\vec{u}(x, t))) \cdot \mathbf{n}=\mathbf{J}_{\vec{u}}^{T} \nabla \psi(\vec{u}) \cdot \mathbf{n}=(\nabla \psi(\vec{u}))^{T} \mathbf{J}_{\vec{u}} \mathbf{n}=(\nabla \psi(\vec{u}))^{T} \mathbf{0}=0$, due to the boundary conditions on the evolution of $\vec{u}$.

We have then obtained that $\nu$ verifies the heat flow with Neumann boundary conditions and with zero initial data. From the uniqueness of the solution, it follows that $\nu(x, t)=0 \forall x \in \mathcal{M}, \forall t \geq 0$.

\footnotetext{
${ }^{8}$ All the calculations that follow don't take into account that $\psi$ might fail to be differentiable at some points. This could be addressed by a regularization argument.
} 


\section{Second Fundamental Form for Implicit Surfaces}

If we compare the gradient descent flow (and Euler-Lagrange equation) we have obtained with the classical one from harmonic maps, we see that the main difference is that the Christoffel symbols of the target manifold term appearing in the classical formulation has been replaced by a new term that includes the Hessian of the embedding function. We obtained this by first embedding the target manifold and then restricting the search for the minimizing map to the class of maps onto the zero level-set of the embedding function. This approach can be followed to apply this framework to any variational problem. We now show how the same equation can be obtained by simply substituting the second fundamental form of the explicit target manifold by the corresponding expression for an implicit target manifold. This will illustrate how to apply our framework to general PDE's, not necessarily gradient descent flow. The basic idea is just to replace all the PDE components concerning the target manifold by their counterparts for implicit representations.

In [28] (page 150) it is shown that the scalar second fundamental form $h$ at a point $p$ of an hypersurface $\mathcal{S}$ can be written in the form

$$
h(p)(V, W)=\frac{\mathbf{H}_{\psi}(p)[V, W]}{\|\nabla \psi\|^{2}}
$$

for $V, W \in T_{p} \mathcal{S}$. According to [28] (page 139) the vectorial second fundamental form is given by

$$
\mathbb{I I}(p)(V, W)=h(p)(V, W) \frac{\nabla \psi}{\|\nabla \psi\|}
$$

From (15) and what we have just seen it is obvious that the implicit version of the harmonic map EulerLagrange is (12).

As stated before, and following the formulas above, the implicit representation of the target surface permits then to compute the second fundamental form using differences on Cartesian grids, without the need to develop new numerical techniques on polygonal grids.

From the result just presented, in order to transform a given PDE into its counterpart when the target manifold is represented in implicit form, all what needs to be done is to re-write all the characteristics of the PDE concerning this target manifold in implicit form. For completeness, in Appendix 1 we present basic facts on calculus on implicitly represented surfaces.

\subsection{Explicit Derivation of the Diffusion Flow}

Here we first proceed in a naïve way to obtain an equivalent formulation of the gradient descent flow that will help in the numerical implementation. We assume we have a family $\{\vec{u}(\vec{x}, t)\}_{t}$ of mappings from $\Omega$ to $\mathcal{N}$. For each $t$ we define the harmonic energy of a member of the family as

$$
E(t)=\frac{1}{2} \int_{\Omega}\left\|\mathbf{J}_{\vec{u}(\vec{x}, t)}\right\|_{F}^{2} d x
$$

We then find a variation of the family such that $E(t)$ decreases. To accomplish this we formally differentiate the energy with respect to $t$. A simple computation yields

$$
\dot{E}(t)=-\int_{\Omega} \vec{u}_{t} \cdot \Delta \vec{u} d x
$$

Now, since $\vec{u}(\vec{x}, t) \in \mathcal{N} \forall \vec{x} \in \Omega$ and $\forall t$ of smooth existence, one must have $\vec{u}_{t}(\vec{x}, t) \in T_{\vec{u}(\vec{x}, t)} \mathcal{N}$. An appropriate choice for $\vec{u}_{t}$ would be

$$
\vec{u}_{t}=\Pi_{T_{\vec{u}(\vec{x}, t)} \mathcal{N}}(\Delta \vec{u})
$$


since this makes $\dot{E}(t)=-\int_{\Omega}\left\|\vec{u}_{t}\right\|^{2} d x \leq 0$.

The projection operator in (22), as we already know (see Appendix 1), can be expressed in a very simple form using $\psi$ (the signed distance function to $\mathcal{N}$ ),

$$
\Pi_{T_{\vec{p}} \mathcal{N}}(\vec{v})=\vec{v}-\vec{v} \cdot \nabla \psi(p) \nabla \psi(p)
$$

Now, it should happen that (22) is equivalent to (16). We show this in $\S 7$.

\subsection{Remarks on the Solutions of the Diffusion Flow}

The well posedness of the diffusion problem with Neumann boundary conditions is addressed in [22, 32], where the following results are obtained, here included for completeness:

Theorem 1 For a given $C^{\infty}$ mapping $\vec{u}_{0}: \mathcal{M} \rightarrow \mathcal{N} \subset \mathbb{R}^{n+1}$ with $\frac{\partial \vec{u}_{0}}{\partial \mathbf{n}}=0$ on $\partial \mathcal{M}$ and for every $2+\operatorname{dim}(\mathcal{M})<p<+\infty$ there exists an $\epsilon>0$ (depending on $\vec{u}_{0}$ ) and a mapping $\vec{u}: \mathcal{M} \rightarrow \mathcal{N}$ of class $\mathbf{L}_{2}^{p}\left(\mathcal{M} \times[0, \epsilon], \mathbb{R}^{n+1}\right) .{ }^{9}$ Moreover, $\vec{u}$ is unique and $C^{\infty}$ except at the corner $\partial \mathcal{M} \times\{0\}$.

Theorem 2 Let $(\mathcal{M}, g)$ and $(\mathcal{N}, h)$ be compact Riemmanian Manifolds with convex boundary. Let $\vec{u}$ : $\mathcal{M} \times[0, \omega) \rightarrow \mathcal{N}$ be a maximal solution of the diffusion problem with initial value a $C^{\infty}$ mapping $\vec{u}_{0},{ }^{10}$ with $\chi_{0} \triangleq\left\|e\left[\vec{u}_{0}\right]\right\|_{\mathbf{L}^{\infty}}>0$. Let $r \in \mathbb{R}$ be such that Ric $\mathcal{M}_{\mathcal{M}} \geq-\frac{r}{2} g,{ }^{11}$ and $R \geq 0$ such that all sectional curvatures of $\mathcal{N}$ are not greater than $\frac{R}{4}$. Then,

1. In the case $r+R \chi_{0}>0$

(a) if $R>0$ then $\left\{\begin{array}{l}\omega \geq \frac{1}{r} \log \left(1+\frac{r}{R \chi_{0}}\right) \text { when } r \neq 0 \\ \omega \geq \frac{1}{R \chi_{0}} \text { when } r=0\end{array}\right.$

(b) if $R=0$ then $\omega=+\infty$.

2. In the case $r+R \chi_{0} \leq 0, \omega=+\infty$.

\section{Maps onto Open Surfaces}

So far, we have only addressed the case when the target surface is closed (zero level-set). In this section we briefly deal with open surfaces. We show that when the function is evolving according to the flow in $\S 2.3$, the set $\mathcal{C}(t) \triangleq\{\vec{u}(x, t), x \in \mathcal{M}\}$ remains inside the initial convex-hull of $\mathcal{C}_{0} \triangleq\left\{\vec{u}_{0}(x), x \in \mathcal{M}\right\}, \forall t \geq 0$. This property is basically a consequence of the maximum principle. Numerically, this might of course be violated due to numerical errors, and we will later discuss how to correct for this as well.

\footnotetext{
${ }^{9} \mathbf{L}_{2}^{p}\left(\mathcal{M} \times[0, \epsilon], \mathbb{R}^{n+1}\right)$ is the space of functions $f: \mathcal{M} \rightarrow \mathbb{R}^{n+1}$ such that for every $i=1, \ldots, n+1, \nabla_{\mathcal{M}} f^{i}, \mathbf{H}_{f^{i}}^{\mathcal{M}}$ and $\frac{\partial f^{i}}{\partial t}$ are all in $\mathbf{L}^{2}(\mathcal{M} \times[0, \epsilon])$.

${ }^{10}$ A solution $\vec{u}: \mathcal{M} \times[0, \omega) \rightarrow \mathcal{N}$ of the diffusion problem is maximal if it cannot be extended to be a solution on $\mathcal{M} \times[0, \omega+\epsilon)$ for any $\epsilon>0$ or if $\omega=+\infty$.

${ }^{11}$ Ric $_{\mathcal{M}}$ stands for the Ricci curvature tensor of $\mathcal{M}$.
} 


\subsection{Motivation: The Planar Case}

Assume that the target manifold $\mathcal{N}$ is flat, for example $R^{k}$ (we still assume that the domain manifold $\mathcal{M}$ is flat). Let $\vec{u}(x, t)$ solve $\frac{\partial \vec{u}}{\partial t}=\Delta \vec{u}$ for $x \in \mathcal{M}$ and $t \geq 0$, and $\left.\frac{\partial \vec{u}}{\partial \mathbf{n}}\right|_{\partial \mathcal{M}}=0$. Let $\Xi$ be a convex set of $R^{k}$ with smooth boundary (this guarantees that the distance function is also smooth almost everywhere, see [36] for a formal statement), and $\xi$ the signed distance function to this set (positive outside and negative inside). Define $g(x, t) \triangleq \xi(\vec{u}(x, t))$. It then immediately follows that $\frac{\partial g}{\partial t}-\Delta g=-\sum_{i=1}^{k} \mathbf{H}_{\xi}\left(\frac{\partial \vec{u}}{\partial x_{k}}, \frac{\partial \vec{u}}{\partial x_{k}}\right) .{ }^{12}$ Since $\Xi$ is convex, so it is $\xi$. Then, the Hessian of $\xi$ is positive semi-definite, meaning that $\frac{\partial g}{\partial t}-\Delta g \leq 0$. Following the scalar maximum principle, $\max _{\{x \in \mathcal{M}, t \geq 0\}} g(x, t)=\max _{\{x \in \mathcal{M}\}} g(x, 0)$. If $\left\{\vec{u}_{0}(x), x \in \mathcal{M}\right\} \subseteq \Xi$, which is equivalent to $0 \geq \xi\left(\vec{u}_{0}(x)\right)=g(x, 0)$, we obtain that $g(x, t) \leq 0$, and $\vec{u}(x, t) \in \Xi$, for all $x \in \mathcal{M}$ $\mathrm{y} t \geq 0$.

\subsection{The General Case}

The main result presented below is from [22]. We quote it here for completeness. ${ }^{13}$

Theorem 3 Let $\vec{u}(x, t)$ be the solution of (16) at time $t$. Let us assume that for $t \leq T$ this solution remains smooth. Let $I_{0}=\vec{u}_{0}(\Omega)$, and $\mathcal{I}_{0}$ be the convex hull of $I_{0}$. Then for $(x, t) \in \Omega \times[0, T], \vec{u}(x, t) \in \mathcal{I}_{0}$.

\section{Maps onto Implicit Submanifolds}

Here we present a modification to the diffusion flow previously presented suited to diffuse data that belongs to a certain submanifold $\mathcal{C}$ of $\mathcal{N}=\{\psi=0\}$. We specify the submanifold by $\{\psi=0\} \cap\{\Phi=0\}$, where we select $\Phi: \mathbb{R}^{N} \rightarrow R$ to be the signed intrinsic (to $\mathcal{N}$ ) distance function to $\{\Phi=0\}$, satisfying (see Appendix 1 for the notation)

$$
1=\left\|\nabla_{\psi} \Phi\right\|=\sqrt{\|\nabla \Phi\|^{2}-|\nabla \psi \cdot \nabla \Phi|^{2}}
$$

In addition we specify the condition

$$
\Phi(p)=0 \text { for } p \in \mathcal{K}_{\Phi}
$$

where

$$
\mathcal{K}_{\Phi}=\left\{x \in \mathbb{R}^{N} \mid x=p+\alpha \nabla \psi(p), \text { with } p \in \mathcal{C}, \alpha \in \mathbb{R}\right\}
$$

is the cone intersecting $\{\psi=0\}$ at $\mathcal{C}$ and director rays normal also to $\{\psi=0\}$.

The reason for specifying the submanifold this way is that we cannot proceed as before, simply specifying the submanifold as the zero level set of it's Euclidean distance function. This is because such function would be singular precisely onto the submanifold.

As we show in Appendix 1, the Hessian of $\Phi$, intrinsic to $\mathcal{N}$ evaluated at the point $p$, and restricted to $T_{p} \mathcal{N}$, can be written in the form

$$
\mathbf{H}_{\Phi}^{\mathcal{N}}(p)=\mathbf{H}_{\Phi}(p)-\Lambda(p) \mathbf{H}_{\psi}(p)
$$

where $\Lambda(p)=\nabla \Phi(p) \cdot \nabla \psi(p)$. This expression will be used below.

\footnotetext{
${ }^{12}$ Note once again that we are omitting details regarding the correct handling of the distance function, since it is not everywhere differentiable. However, by a regularization argument, the same conclusion holds.

${ }^{13}$ The proof of this result has a lot of interest in itself since it can be carried out within the implicit framework introduced in this paper.
} 


\subsection{The Minimization of the Functional}

We now derive the Euler-Lagrange corresponding to this additional mapping restriction. For this, we use a technique slightly different that the one in $\S 2.1$.

Let us assume that $\vec{u}$ achieves a minimum of the energy functional (1). We must build a variation of $\vec{u}$ that belongs to $\mathcal{C}$, the intersection of the zero level-sets of two embedding functions (and not just of $\psi$ as before). It is clear that one such variation would be

$$
\vec{w}_{\lambda}=\Pi_{\mathcal{C}}(\vec{u}+\lambda \vec{v})
$$

We are interested only on those terms of $e\left[\vec{w}_{\lambda}\right]$ that do not vanish after the $\left.\sum_{i=1}^{N} \frac{\partial}{\partial x_{i}}(\bullet)\right|_{\lambda=0}$ operation, namely those linear in $\lambda$. Therefore we only preserve those terms in $\vec{w}_{\lambda}$ which are constant or linear in $\lambda$ :

$$
\vec{w}_{\lambda} \simeq \vec{u}+\lambda \Pi_{T_{\vec{u}} \mathcal{C}}(\vec{v})
$$

We write

$$
\begin{aligned}
\Pi_{T_{\vec{u}} \mathcal{C}}(\vec{v}) & =\Pi_{T_{\vec{u}}\{\psi=0\}}\left\{\vec{v}-\left(\vec{v} \cdot \nabla_{\psi} \Phi(\vec{u})\right) \nabla_{\psi} \Phi(\vec{u})\right\} \\
& =\vec{v}-\left(\vec{v} \cdot \nabla_{\psi} \Phi(\vec{u})\right) \nabla_{\psi} \Phi(\vec{u})-(\vec{v} \cdot \nabla \psi(\vec{u})) \nabla \psi(\vec{u})
\end{aligned}
$$

where $\nabla_{\psi} \Phi(\vec{u})=\nabla \Phi(\vec{u})-\Lambda(\vec{u}) \nabla \psi(\vec{u})$ is the gradient of $\Phi$ intrinsic to $\{\psi=0\}$.

In this way we find that (up to a first order in $\lambda$ ):

$$
\begin{aligned}
e\left[\vec{w}_{\lambda}\right] & \simeq e[\vec{u}]+\lambda \sum_{i=1}^{N} \vec{u}_{x_{i}} \cdot\left[\vec{v}_{x_{i}}-\vec{v}_{x_{i}} \cdot \nabla_{\psi} \Phi(\vec{u}) \nabla_{\psi} \Phi(\vec{u})\right. \\
& -\vec{v} \cdot \frac{\partial \nabla_{\psi} \Phi(\vec{u})}{\partial x_{i}} \nabla_{\psi} \Phi(\vec{u})-\vec{v} \cdot \nabla_{\psi} \Phi(\vec{u}) \frac{\partial \nabla_{\psi} \Phi(\vec{u})}{\partial x_{i}} \\
& \left.-\vec{v}_{x_{i}} \cdot \nabla \psi(\vec{u}) \nabla \psi(\vec{u})-\vec{v} \cdot \frac{\partial \nabla \psi(\vec{u})}{\partial x_{i}} \nabla \psi(\vec{u})-\vec{v} \cdot \nabla \psi(\vec{u}) \frac{\partial \nabla \psi(\vec{u})}{\partial x_{i}}\right]
\end{aligned}
$$

Since $\Phi(\vec{u})=\psi(\vec{u})=0$, differentiating with respect to $x_{i}$ we obtain that $\nabla \Phi(\vec{u}) \cdot \vec{u}_{x_{i}}=\nabla \psi(\vec{u}) \cdot \vec{u}_{x_{i}}=0$, and therefore

$$
\nabla_{\psi} \Phi(\vec{u}) \cdot \vec{u}_{x_{i}}=0
$$

The expression (25) can be simplified to obtain

$$
e\left[\vec{w}_{\lambda}\right] \simeq e[\vec{u}]+\lambda \sum_{i=1}^{N} \vec{u}_{x_{i}} \cdot\left[\vec{v}_{x_{i}}-\vec{v} \cdot \nabla_{\psi} \Phi(\vec{u}) \frac{\partial \nabla_{\psi} \Phi(\vec{u})}{\partial x_{i}}-\vec{v} \cdot \nabla \psi(\vec{u}) \frac{\partial \nabla \psi(\vec{u})}{\partial x_{i}}\right]
$$

Moreover, since

$$
\frac{\partial \nabla_{\psi} \Phi(\vec{u})}{\partial x_{i}}=\mathbf{H}_{\Phi} \vec{u}_{x_{i}}-\frac{\partial \Lambda}{\partial x_{i}}(\vec{u}) \nabla \psi(\vec{u})-\Lambda(\vec{u}) \mathbf{H}_{\psi} \vec{u}_{x_{i}}
$$

we have

$$
\frac{\partial \nabla_{\psi} \Phi(\vec{u})}{\partial x_{i}} \cdot \vec{u}_{x_{i}}=\mathbf{H}_{\Phi}^{\mathcal{N}}\left[\vec{u}_{x_{i}}, \vec{u}_{x_{i}}\right]
$$


With all this in mind we find that (again, up to first order in $\lambda$ )

$$
e\left[\vec{w}_{\lambda}\right] \simeq e[\vec{u}]+\lambda \sum_{i=1}^{N} \vec{u}_{x_{i}} \cdot\left[\vec{v}_{x_{i}}-\vec{v} \cdot \nabla_{\psi} \Phi(\vec{u}) \mathbf{H}_{\Phi}^{\mathcal{N}}\left[\vec{u}_{x_{i}}, \vec{u}_{x_{i}}\right]-\vec{v} \cdot \nabla \psi(\vec{u}) \mathbf{H}_{\psi}(\vec{u})\left[\vec{u}_{x_{i}}, \vec{u}_{x_{i}}\right]\right]
$$

Using this expression, after imposing that $\left.\frac{\partial}{\partial \lambda}\right|_{\lambda=0} \int_{\Omega} e\left[\vec{w}_{\lambda}\right] d v=0$ for every $\vec{v}$, we find that the EulerLagrange is

$$
\Delta \vec{u}+\left(\sum_{k} \mathbf{H}_{\psi}\left[\frac{\partial \vec{u}}{\partial x_{k}}, \frac{\partial \vec{u}}{\partial x_{k}}\right]\right) \nabla \psi(\vec{u})+\left(\sum_{k} \mathbf{H}_{\Phi}^{\mathcal{N}}\left[\frac{\partial \vec{u}}{\partial x_{k}}, \frac{\partial \vec{u}}{\partial x_{k}}\right]\right) \nabla_{\psi} \Phi(\vec{u})=0 .
$$

an expression utterly predictable.

\subsubsection{Simple Verification}

As for the case of closed manifolds, we now verify that in fact the gradient descent corresponding to the Euler-Lagrange (26) keeps $\vec{u}$ in $\{\psi=0\} \cap\{\Phi=0\}$. We just need to show that $\nu(x, t) \triangleq \psi(\vec{u}(x, t))$ and $\mu(x, t) \triangleq \Phi(\vec{u}(x, t))$ are always zero. The idea is the same we used in $\S 2.4$, it is enough to show that both $\nu$ and $\mu$ satisfy the heat equation with adiabatic boundary conditions.

1. $\psi$

We have

$$
\nu_{t}=\nabla \psi \cdot \Delta \vec{u}+\sum_{k} \mathbf{H}_{\psi}\left[\frac{\partial \vec{u}}{\partial x_{k}}, \frac{\partial \vec{u}}{\partial x_{k}}\right]
$$

since $\nabla \psi \perp \nabla_{\psi} \Phi$. Also

$$
\Delta \nu=\nabla \psi \cdot \Delta \vec{u}+\sum_{k} \mathbf{H}_{\psi}\left[\frac{\partial \vec{u}}{\partial x_{k}}, \frac{\partial \vec{u}}{\partial x_{k}}\right]
$$

and

$$
\nu_{t}=\Delta \nu
$$

2. $\Phi$

We have

$$
\mu_{t}=\nabla \Phi \cdot \Delta \vec{u}+\nabla \Phi \cdot \nabla_{\psi} \Phi\left(\sum_{k} \mathbf{H}_{\Phi}^{\mathcal{N}}\left[\frac{\partial \vec{u}}{\partial x_{k}}, \frac{\partial \vec{u}}{\partial x_{k}}\right]\right)+\Lambda\left(\sum_{k} \mathbf{H}_{\psi}\left[\frac{\partial \vec{u}}{\partial x_{k}}, \frac{\partial \vec{u}}{\partial x_{k}}\right]\right)
$$

From $\nabla \Phi \cdot \nabla_{\psi} \Phi=\nabla_{\psi} \Phi \cdot \nabla_{\psi} \Phi=\left\|\nabla_{\psi} \Phi\right\|^{2}=1$, the above equation continues as 


$$
\begin{aligned}
& =\nabla \Phi \cdot \Delta \vec{u}+\left(\sum_{k} \mathbf{H}_{\Phi}^{\mathcal{N}}\left[\frac{\partial \vec{u}}{\partial x_{k}}, \frac{\partial \vec{u}}{\partial x_{k}}\right]\right)+\Lambda\left(\sum_{k} \mathbf{H}_{\psi}\left[\frac{\partial \vec{u}}{\partial x_{k}}, \frac{\partial \vec{u}}{\partial x_{k}}\right]\right) \\
& =\nabla \Phi \cdot \Delta \vec{u}+\left(\sum_{k} \mathbf{H}_{\Phi}\left[\frac{\partial \vec{u}}{\partial x_{k}}, \frac{\partial \vec{u}}{\partial x_{k}}\right]\right)
\end{aligned}
$$

Also

$$
\Delta \mu=\nabla \Phi \cdot \Delta \vec{u}+\left(\sum_{k} \mathbf{H}_{\Phi}\left[\frac{\partial \vec{u}}{\partial x_{k}}, \frac{\partial \vec{u}}{\partial x_{k}}\right]\right)
$$

and then

$$
\mu_{t}=\Delta \mu
$$

Finally, it is easy to see that both $\nu$ and $\mu$ satisfy Neumann boundary conditions. Since at $t=0$ both functions are zero, we must have that they are identically zero.

\section{Implicit Domain Manifolds and $p$-Harmonic Maps}

For completeness, we present now the formulas corresponding to the case where both the domain and target manifolds are represented in implicit form (with the implicitizing functions being the corresponding signed distance ones). Deriving these formulas is straightforward using the framework here presented, when combined with the work in [3]. We also show the corresponding flows for $p$-harmonic maps.

\section{$5.1 p$-Harmonic Maps}

We still assume $\mathcal{M}$ to be planar. The energy density (2) (but no the dependence of the energy on its density) is redefined as follows. For every $p \in[1,+\infty)$ let

$$
e_{p}[\vec{u}] \triangleq \frac{1}{p}\left\|\mathbf{J}_{\vec{u}}\right\|_{\mathcal{F}}^{p}
$$

A simple application of variational calculus leads to conclude that ${ }^{14}$

$$
\vec{u}_{t}=p^{1-\frac{2}{p}} \mathbf{\Pi}_{\nabla \psi(\vec{u})}\left(\nabla \cdot\left(\left(e_{p}[\vec{u}]\right)^{1-\frac{2}{p}} \mathbf{J}_{\vec{u}}^{T}\right)\right)
$$

Note that if $p<2$ difficulties are expected to arise, see [40] and the references therein.

\subsection{Generic (Implicit) Domain Manifolds}

Let $\mathcal{M}=\left\{x \in \mathbb{R}^{m}: \phi(x)=0\right\}$, where $\phi(\cdot)$ is the signed distance function to $\mathcal{M}$, then the diffusion is given by:

$$
\vec{u}_{t}=\nabla \cdot\left(\mathbf{\Pi}_{\nabla \phi} \mathbf{J}_{\vec{u}}^{T}\right)+\left(\sum_{k, r} \mathbf{H}_{\psi}\left[\vec{u}_{x_{r}}, \vec{u}_{x_{k}}\right]\left(\boldsymbol{\Pi}_{\nabla \phi}\right)_{k r}\right) \nabla \psi
$$

\footnotetext{
${ }^{14}$ The divergence operator convention (for a matrix $A$ ) we have used is $\nabla \cdot A=\left(\nabla \cdot \overrightarrow{A_{v 1}}|\ldots| \nabla \cdot \overrightarrow{A_{v r}}\right.$ ), where $\overrightarrow{A_{v i}}$ stands for the $i$-th column of $A$. That is, we apply a columnwise divergence.
} 
The whole deduction rests upon the redefinition of the energy (1) and its density (2). Now we should define the energy density to be

$$
e_{\phi}[\vec{u}] \triangleq \frac{1}{2}\left\|\mathbf{J}_{\vec{u}}^{\phi}\right\|_{\mathcal{F}}^{2}
$$

where the intrinsic Jacobian of $\vec{u}$ can be written as (see Appendix 1 for more details) $\mathbf{J}_{\vec{u}}^{\phi}=\mathbf{J}_{\vec{u}} \mathbf{\Pi}_{\nabla \phi}$.

The new definition for the energy should be: ${ }^{15}$

$$
E[\vec{u}] \triangleq \int_{\mathbb{R}^{m}} e_{\phi}[\vec{u}] \delta(\phi(x)) d x
$$

Comparing this with (15), we can infer the implicit form of the Christoffel symbols: ${ }^{16}$

$$
\boldsymbol{\Gamma}_{i j}^{l}(\vec{u})=\frac{\partial^{2} \psi}{\partial u^{i} \partial u^{j}}(\vec{u}) \frac{\partial \psi}{\partial u^{l}}(\vec{u})
$$

\subsection{Generic (Implicit) Domain Manifold and $p$-Harmonic Maps}

Using both generalization presented above, we arrive at the following formula with a bit more computational effort

$$
\vec{u}_{t}=p^{1-\frac{2}{p}} \mathbf{\Pi}_{\nabla \psi(\vec{u})}\left(\nabla \cdot\left(\left(e_{\phi, p}[\vec{u}]\right)^{1-\frac{2}{p}} \Pi_{\nabla \phi} \mathbf{J}_{\vec{u}}^{T}\right)\right)
$$

where

$$
e_{\phi, p}[\vec{u}] \triangleq \frac{1}{p}\left\|\mathbf{J}_{\vec{u}}^{\phi}\right\|_{\mathcal{F}}^{p}
$$

\section{Diffusion of Tangent and Normal Directions}

Throughout this section we will assume $\operatorname{dim}(\mathcal{M})=\operatorname{dim}(\mathcal{N})$. Assume we want to diffuse intrinsic vectorial data constrained to be a direction (unit norm) and to be either normal or tangent to the domain manifold. We can then minimize the functional (29) taking a variation of the form (assume $\vec{u}$ minimizes the energy functional while satisfying both $\|\vec{u}\|=1$ and $\Pi(\vec{u})=\vec{u})$

$$
\vec{u}_{\lambda}(x) \triangleq \frac{\vec{u}+\lambda \Pi(\vec{v})}{\|\vec{u}+\lambda \Pi(\vec{v})\|}
$$

where $\vec{v}: \mathcal{M} \rightarrow \mathbb{R}^{d}$ is smooth and $\Pi$ is either $\boldsymbol{\Pi}_{T_{x} \mathcal{M}}$ or $\boldsymbol{\Pi}_{N_{x} \mathcal{M}}$ (projection onto the tangent or normal space respectively). Let $\vec{w}=\Pi(\vec{v})$, then it follows easily that

$$
\left.\frac{d E\left[\vec{u}_{\lambda}\right]}{d t}\right|_{\lambda=0}=-\int_{\mathbb{R}^{m}}\left\{\Delta_{\phi} \vec{u}+2 e_{\phi}[\vec{u}] \vec{u}\right\} \cdot \vec{w} \delta(\phi(x)) d x
$$

Imposing $\left.\frac{d E\left[\vec{u}_{\lambda}\right]}{d t}\right|_{\lambda=0}=0$ for all $v$ implies

$$
\Pi\left(\Delta_{\phi} \vec{u}+2 e_{\phi}[\vec{u}] \vec{u}\right)=\Pi\left(\Delta_{\phi} \vec{u}\right)+2 e_{\phi}[\vec{u}] \vec{u}=0
$$

\footnotetext{
${ }^{15}$ We have already taken into account that $\|\nabla \phi\|=1$.

${ }^{16}$ Of course $g^{i j}=\left(\Pi_{\nabla \phi}\right)_{i j}\left(=g_{i j}^{-1}\right)$. Then, it is nice to observe (although formally incorrect) that since $\boldsymbol{\Pi}_{\nabla \phi} \nabla \phi=0$, then the metric $g: \mathbb{R}^{d} \rightarrow \mathbb{R}^{d \times d}$ has eigenvalue $+\infty$ in the direction given by $\nabla \phi$ thus prohibiting intermingling of information between adjacent level sets of $\phi$.
} 
Finally, the diffusion flow obtained is

$$
\frac{\partial \vec{u}}{\partial t}(x, t)=\Pi_{x}\left(\Delta_{\phi} \vec{u}(x, t)\right)+2 e_{\phi}[\vec{u}](x, t) \vec{u}(x, t)
$$

Note that if the PDE (31) admits a smooth solution until time $T$, and if (for instance) we are dealing with tangent directions diffusion, the function $f(x, t) \triangleq \nabla \phi(x) \cdot \vec{u}(x, t)$ satisfies $f_{t}(x, t)=2 e_{\phi}[\vec{u}] f(x, t)$. Therefore

$$
f(x, t)=f(x, 0) e^{2 \int_{0}^{t} e[\vec{u}](x, t) d t}
$$

thus verifying that if $\nabla \phi(x) \cdot \vec{u}(x, 0)=0$ then $\nabla \phi(x) \cdot \vec{u}(x, t)=0$ for $t \leq T$. We also want to check whether $\|\vec{u}(x, t)\|=1 \forall(x, t)$. Let

$$
F_{\phi}[\vec{u}](t) \triangleq \frac{1}{2} \int_{\mathbb{R}^{d}}\left\|\mathbf{J}_{\vec{u}}\right\|_{\mathcal{F}}^{2} \delta(\phi(x)) d x
$$

then $\dot{F}_{\phi}[\vec{u}](t)=-\int_{\mathbb{R}^{d}} \vec{u}_{t} \cdot \Delta_{\phi} \vec{u} \delta(\phi(x)) d x$. Since both $\|\vec{u}\|=1$ and $\Pi(\vec{u})=\vec{u}$ (so $\Pi\left(\vec{u}_{t}\right)=\vec{u}_{t}$ since $\Pi$ does not depend on $t$ ) must hold, and in order to make $\dot{F}_{\phi}[\vec{u}](t)$ non-positive we choose

$$
\vec{u}_{t}=\Pi \Pi_{T_{\vec{u}}\{\|\vec{u}\|=c\}} \Delta_{\phi} \vec{u}
$$

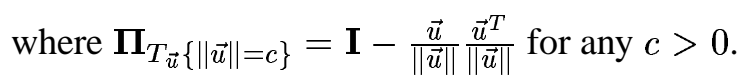

Note that the above evolution indeed forces $\vec{u}(x, t)$ to satisfy both imposed conditions. Let $\vec{v}: \mathbb{R}^{d} \rightarrow$

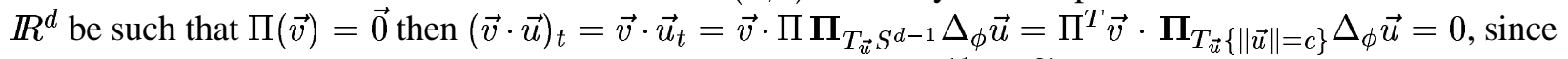
the projection matrix is symmetric, and just using this we have $\left(\frac{1}{2}\|\vec{u}\|^{2}\right)_{t}=\vec{u} \cdot \vec{u}_{t}=\vec{u} \cdot \mathbf{\Pi}_{T_{\vec{u}}\{\|\vec{u}\|=c\}}=0$ trivially. Finally, using $\|\vec{u}\|=1$ and carrying out some computations in a way similar to $\S 7$ below, ${ }^{17}$ one can prove that (32) reduces to (31).

\section{Numerical Implementation}

We now discuss the numerical implementation of the flows previously introduced. Since the target manifold is now implicitly represented, we can basically use classical numerical techniques on Cartesian grids. Although as we have shown, the flows guarantee that the map remains on the target (sub-)manifold, numerical errors can move it away from it, requiring a simple projection step.

When dealing with submanifolds, although the evolution equations also guarantee that the solution will remain inside the convex hull, once again due to numerical discretization $\vec{u}$ could be taken outside of it during the evolution. In order to numerically project it back, we need to have a distance function to this convex hull defined on the implicitly defined target manifold. In [30] we have shown how to computationally optimal compute such a distance function on implicitly defined manifolds, and this is the technique used for this projection into the convex hull.

An explicit scheme can be devised to implement (28). However it turns out that it is more convenient to implement a mathematically equivalent evolution, as shown in [12]. More specifically, the equivalent evolution is

$$
\frac{\partial u}{\partial t}=\Delta u-(\Delta u \cdot \nabla \psi) \nabla \psi
$$

That both evolutions are equivalent is easy to see, and we show this next.

\footnotetext{
${ }^{17}$ The main difference is that now one must take into account the Laplace-Beltrami expressed "implicitly," see Appendix 1 for more details on intrinsic differential operators within the implicit framework.
} 
One has that $f(x, t) \triangleq \psi(\vec{u}(x, t))=0 \forall(x, t) \in \Omega \times \mathbb{R}^{+} \cup\{0\}$ for $\vec{u}(\cdot, \cdot)$ satisfying (16). Now, differentiating $f$ with respect to $x_{i}$ we obtain

$$
\nabla \psi(\vec{u}) \cdot \vec{u}_{x_{i}}=0
$$

Differentiating again with respect to $x_{i}$,

$$
\mathbf{H}_{\psi}\left[\vec{u}_{x_{i}}, \vec{u}_{x_{i}}\right]+\nabla \psi \cdot \vec{u}_{x_{i} x_{i}}=0
$$

Summing for all $i$,

$$
\sum_{i} \mathbf{H}_{\psi}\left[\vec{u}_{x_{i}}, \vec{u}_{x_{i}}\right]+\nabla \psi \cdot \Delta \vec{u}=0
$$

and using the previous expression we derive (33) from (16).

\subsection{Numerical Scheme}

All the coding was done using Flujos as the main core (see [19]) and VTK (see [49]) for visualization purposes. All the examples below were carried based in equation (30). Its numerical implementation is straightforward (at least when $p=2$ ). We used forward time discretization (explicit scheme), and for the spatial discretization, we used the following well known recipe. To spatially discretize

$$
f_{t}(x, t)=\nabla \cdot(\mathbf{K}(x) \nabla f(x, t))
$$

$(\mathbf{K}(x)$ is a symmetric positive semi-definite matrix), we consider backward approximation of the divergence and a forward approximation of the gradient. Let's explain how this applies in our situation, and for that we assume $p=2$ in (30). Then the equation we have to implement is

$$
\vec{u}_{t}(x, t)=\mathbf{\Pi}_{\nabla \psi(\vec{u}(x, t))}\left(\nabla \cdot\left(\mathbf{\Pi}_{\nabla \phi(x)}\left(\mathbf{J}_{\vec{u}}^{T}(x, t)\right)\right)\right)
$$

If we don't take into account the outer projection matrix, every coordinate of $\vec{u}$ evolves according to

$$
u_{t}^{i}(x, t)=\nabla \cdot\left(\mathbf{\Pi}_{\nabla \phi(x)} \nabla u^{i}(x, t)\right)
$$

having for each component the same structure than the model evolution (34). We then borrow the above discretization for our evolution. If we consider the coupling among different $u$ 's imposed by the projection matrix $\boldsymbol{\Pi}_{\nabla \psi(\cdot)}$, we see that we still preserve numerical stability since this matrix is positive semidefinite and has spectral radius not greater than $1 .{ }^{18}$ In more detail, it can be shown after some calculations (see [21, 42]) that for the scheme ( $p$ now denotes a position over the grid)

$$
\vec{v}_{p}^{n+1}=\vec{v}_{p}^{n}+\Delta t \mathbf{P}\left(\vec{v}_{p}^{n}\right)\left(\nabla^{-} \cdot\left(\mathbf{Q}(p) \nabla^{+} \vec{v}_{p}^{n}\right)\right)
$$

the stability condition is of the form $\left(\lambda=\frac{\Delta t}{(\Delta x)^{2}}\right)$

$$
\lambda \leq \min _{p, u} \frac{S(p)}{\rho(\mathbf{P}(u)) \max \left\{S^{2}(p), D^{2}(p)\right\}}
$$

or

\footnotetext{
${ }^{18}$ Note that $\|\vec{v}\|^{2} \geq \Pi_{\nabla \psi}[\vec{v}, \vec{v}]=\|v\|^{2}-|\nabla \psi \cdot \vec{v}|^{2} \geq 0$ for all $\vec{v}$. We have used that $\phi$ is a distance function.
} 


$$
\lambda \leq \frac{1}{\max _{u} \rho(\mathbf{P}(u))} \min _{p}\left\{\frac{S(p)}{\max \left\{S^{2}(p), D^{2}(p)\right\}}\right\}
$$

where $\rho(\mathbf{P}(p))$ stands for the spectral radius of the matrix $\mathbf{P}(p), S(p)=\sum_{i j}\left(q_{i j}(p)+q_{i j}\left(p-\Delta x \vec{e}_{i}\right)\right)$, and $D(p)=\sum_{i j}\left(q_{i j}(p)-q_{i j}\left(p-\Delta x \vec{e}_{i}\right)\right)$. In our case we may admit $D(\cdot)$ to be small compared with $S(\cdot)$ (given the identification $\mathbf{Q} \leftrightarrow \boldsymbol{\Pi}_{\nabla \phi}$ ) when $\Delta x$ is small. This can be easily related to the curvatures of $\{\phi=0\}$ giving a condition on the sampling of the distance function $(\phi)$ representing the domain manifold. This condition mainly means that we require a fine enough sampling as to guarantee that the change in the normals to the level surfaces of $\phi$ is small between adjacent grid points. This condition is obviated when the domain manifold is planar. So the stability condition becomes

$$
\lambda \leq \frac{1}{\max _{u} \rho(\mathbf{P}(u)) \max _{p} S(p)}
$$

Since by Cauchy-Schwartz's inequality (and the aforementioned assumption on the change of $\nabla \phi$ between adjacent grid points) $2 d$ (in practise) upper-bounds $S(p)$, remembering the fact that $\rho(\mathbf{P}(p)) \leq 1$, we arrive at $\lambda \leq \frac{1}{2 d}$. Note that if a more careful implementation is desired, good choices are ADI or AOS schemes, see $[50]$.

All derivatives in $\boldsymbol{\Pi}_{\nabla \psi(\cdot)}$ and $\boldsymbol{\Pi}_{\nabla \phi(\cdot)}$ were approximated by central differences. An interpolation scheme had to be used since the evaluations of $\boldsymbol{\Pi}_{\nabla \psi(\cdot)}$ in the above equation are at positions given by $\vec{u}(x, t)$, positions not necessarily on the underlying grid. We used linear interpolation for this purpose.

Note that as done in [3], when the domain manifold is also implicitly represented, the values of the map on it are periodically extended to its surrounding offset due to stability considerations. Also, as explained before, due to numerical discretization, the discretely computed solution map can be taken out of the target manifold during the evolution. In this paper, we simply project it back at every iteration. We have seen that this projection is a trivial step due to the fact that the embedding is a distance function. It is quite straightforward to show that the results reported in [1] can be extended for our equations as well, at least for convex hyper-surfaces.

\subsection{Numerical Examples}

In all the examples below, the domain manifold $\mathcal{M}$ is either the Euclidean space $\mathbb{R}^{2}$ or an implicit torus. The target manifold $\mathcal{N}$ is an implicit surface in $\mathbb{R}^{3}$, that is, the zero level-set of $\psi: \mathbb{R}^{3} \rightarrow \mathbb{R}, \psi$ being a signed distance function (this is of course also the case when the surface is a sphere, $\psi$ being as in $\S 2.4$ ).

In order to present interesting examples we construct texture maps, add noise to them, and then diffuse them using our framework. Let $\mathcal{S}$ be the surface onto which we want to map a given (planar) image defined in a subset $D \subset \mathbb{R}^{2}$. Then the texture pap is a map $\vec{T}: \mathcal{S} \rightarrow D$. Once the map is known, we inverted it to find a map $\vec{u}_{0}: D \rightarrow \mathcal{S}$. Then, we built up the noisy map $\vec{u}: D \rightarrow \mathcal{S}$ defined by

$$
\vec{u}(x)=\boldsymbol{\Pi}_{\mathcal{S}}\left(\vec{u}_{0}(x)+\overrightarrow{\mathbf{n}}(x)\right)
$$

where $\overrightarrow{\mathbf{n}}: D \rightarrow \mathcal{S}$ is random map with small prescribed power $\sigma$. We then feed the evolution (16) with $\vec{u}$ as initial condition, and Neumann boundary conditions. After a certain number of steps we stop the evolution, invert the resulting map, and use it as a texture map to paint the surface with a certain texture. ${ }^{19}$

As a means of finding a suitable $\vec{T}$ we have extended the work in [47] (a multidimensional scaling approach), combined with the technique developed in [30] for computing distances on implicit surfaces.

\footnotetext{
${ }^{19}$ Note that we are not proposing this as a complete texture mapping alternative, it is just to provide an illustrative example.
} 

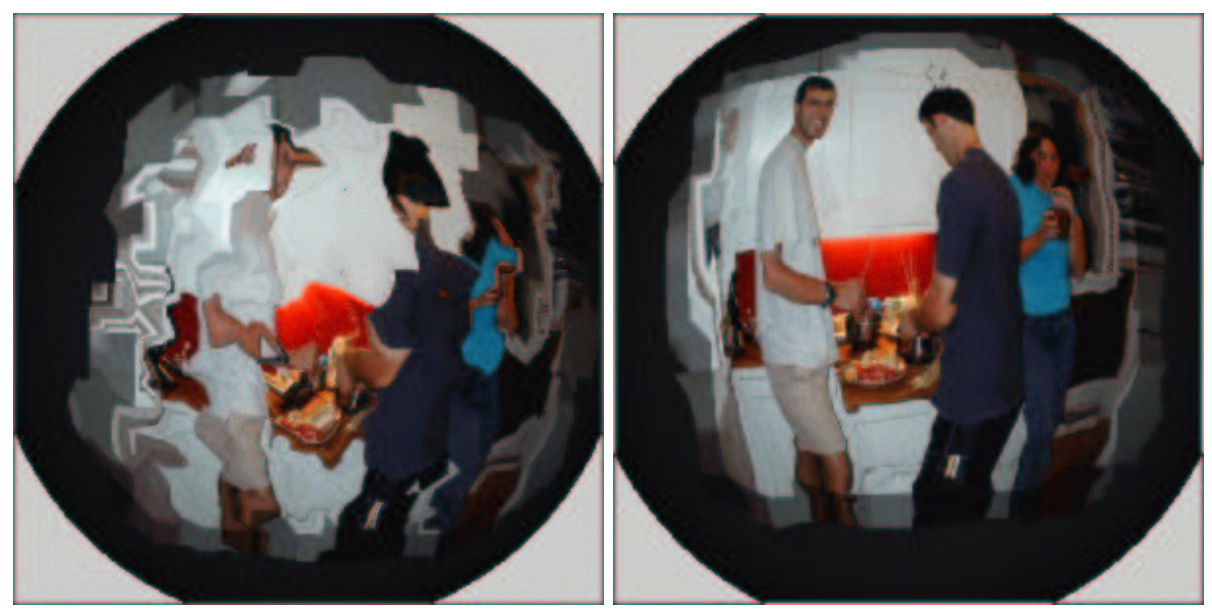

Figure 1: Diffusion of a noisy texture map (left) onto an implicit sphere (right). (This is a color figure.)

In all the steps just described there are some minor implementation details, mainly regarding interpolation tasks, that we omit for the sake of clarity.

In Figures 1, 2 and 3 we then denoise vectors from the plane $\mathbb{R}^{2}$ to a 3D surface defined as the zero level-set of $\psi: \mathbb{R}^{3} \rightarrow \mathbb{R}$ and map a texture image to the surface using the obtained map. Note that the map is the one being processed, not the image itself.

We also show an example of diffusion of random maps from an implicit torus to the implicit bunny model, see Figure 4. As expected from the theory, when evolving this set with the harmonic flow, the set converges to a unique point.

\section{Conclusions}

In this paper we have shown how to implement variational problems and partial differential equations onto general target surfaces. We have also addressed the case of open target surfaces and sub-manifolds. The key concept is to represent the target (sub-)manifolds in implicit form, and then implement the equations in the corresponding embedding space. This framework completes the work with general domain manifolds reported in [3], thereby providing a complete solution to the computation of maps between generic manifolds.

\section{Acknowledgments}

We thank Alberto Bartesaghi, Marcelo Bertalmio and Robert Gulliver for interesting conversations during this work and also Alvaro Pardo for his careful reading of the paper. FM performed part of this work while visiting the University of Minnesota. This work was partially supported by a grant from the Office of Naval Research ONR-N00014-97-1-0509, the Office of Naval Research Young Investigator Award, the Presidential Early Career Awards for Scientists and Engineers (PECASE), a National Science Foundation CAREER Award, and the National Science Foundation Learning and Intelligent Systems Program (LIS). 

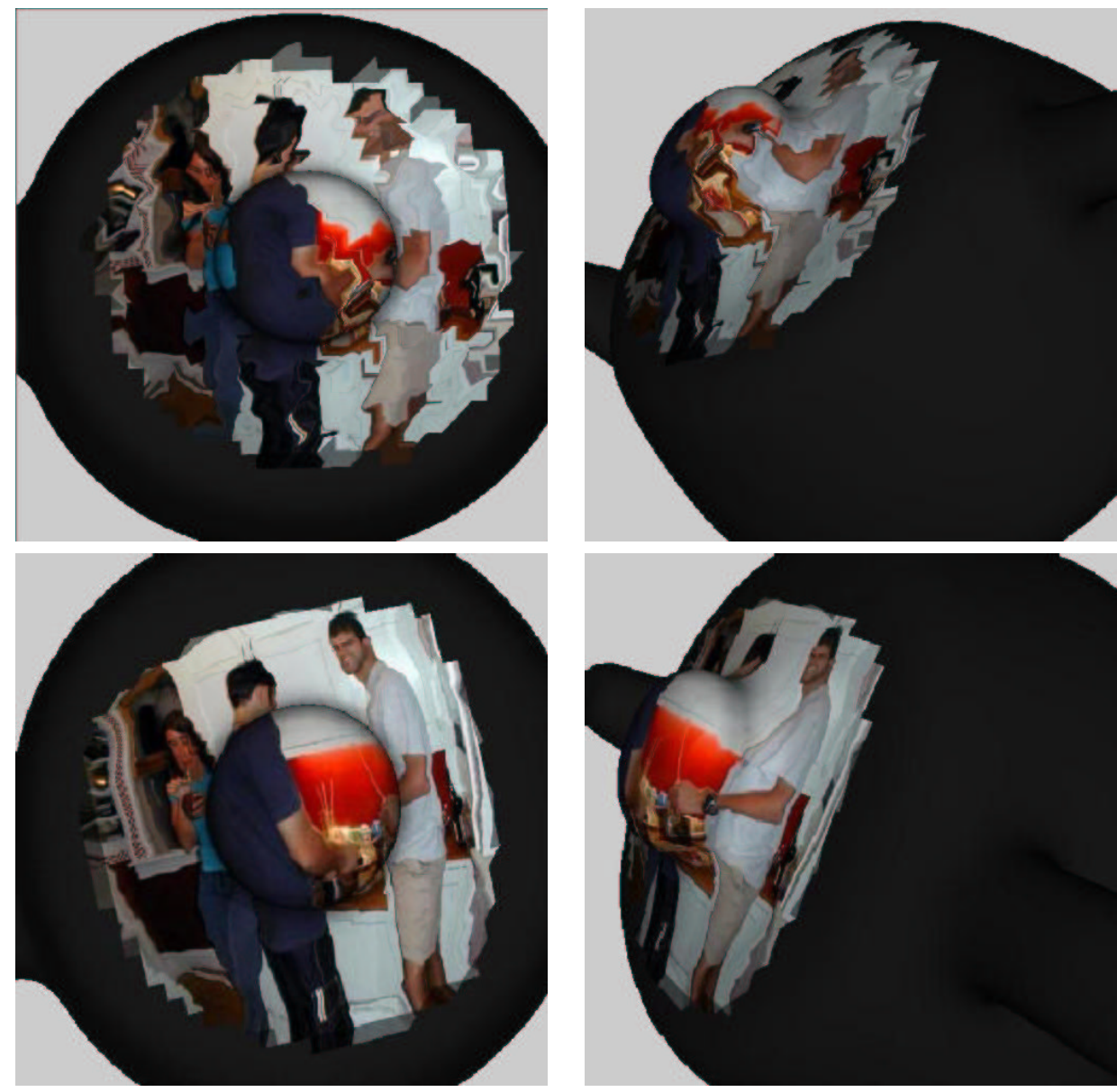

Figure 2: Diffusion of a noisy texture map onto an implicit teapot. We show two different views (noisy on the top and regularized on the bottom). (This is a color figure.) 

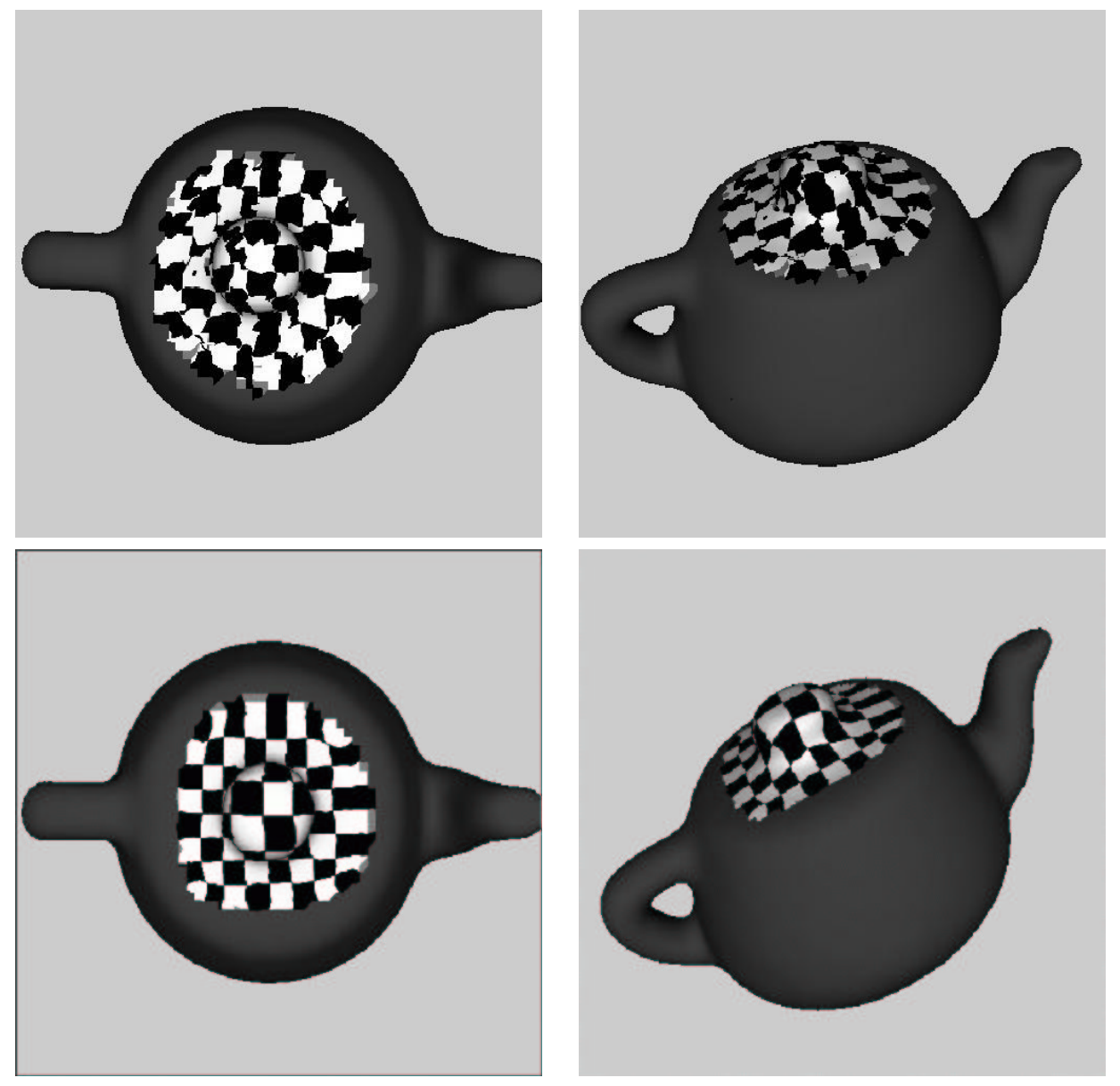

Figure 3: Diffusion of a texture map for an implicit teapot (noisy on he top and regularized on the bottom). A chess board texture is mapped. (This is a color figure.) 

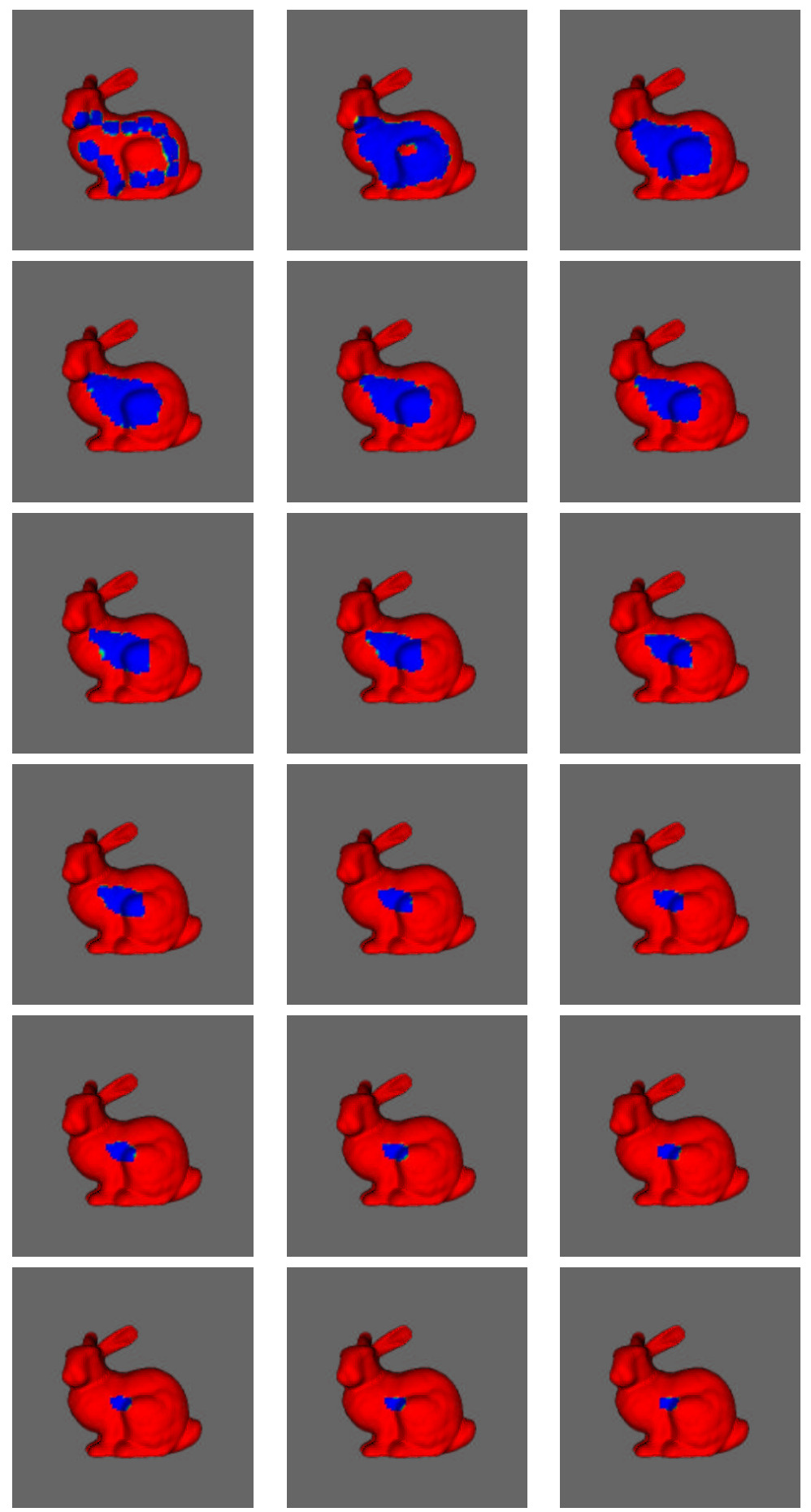

Figure 4: Diffusion of a random map from an implicit torus to the implicit bunny. In blue are marked those points of the bunny's surface pointed by the map at every instant. Different figures correspond to increasing instances of the evolution, from top to bottom and left to tight. We show the map at 17 of 100 iterations performed to the initial map with a time step of .01. We used the 2 -harmonic heat flow with adiabatic conditions. (This is a color figure.) 


\section{Appendix 1: Implicit Calculus}

We now present basic facts about differential calculus on implicitly represented surfaces. For more information see for example [2, 8, 29].

We have a smooth scalar function $f: \mathbb{R}^{d} \rightarrow \mathbb{R}$, and a smooth vector field $\vec{\lambda}: \mathbb{R}^{d} \rightarrow \mathbb{R}^{D}$ ( $d$ and $D$ are not necessarily equal). The manifold onto which the calculus is to be done is represented as $\mathcal{S}=\{\psi=0\}$, for $\psi(\cdot)$ the signed distance function to $\mathcal{S}$.

All the ideas of differentiation can be obtained from simple considerations related to the restriction of the function to a geodesic curve living in the manifold. We consider an arc-length parameterized geodesic curve $\gamma:[-\epsilon, \epsilon] \rightarrow \mathcal{S}$ such that $\gamma(0)=p$ is a given point of $\mathcal{S}$. We denote $F(t)=f(\gamma(t))$ and $\vec{\Lambda}(t)=\vec{\lambda}(\gamma(t))$.

\section{Implicit gradient}

We differentiate once $F(t)$ to obtain $\dot{F}(0)=\nabla f(p) \cdot \dot{\gamma}(0)$. Since $\dot{\gamma}(0) \in T_{p} \mathcal{S}$ (the tangent plane), we find the implicit gradient of $f$ at $p$ to be $\nabla_{\mathcal{S}} f(p)=\nabla f(p)-\nabla f(p) \cdot \vec{n}(p) \vec{n}(p)$, where $\vec{n}(p)$ stands for the normal to the manifold at $p$. Since we can also write $\vec{n}(p)=\nabla \psi(p)$, we obtain

$$
\nabla_{\mathcal{S}} f(p) \triangleq \nabla f(p)-(\nabla f(p) \cdot \nabla \psi(p)) \nabla \psi(p)
$$

We often use the alternative notation $\nabla \psi f$ since the definition can be applied to any level set of $\psi$. Note that we can write $\nabla_{\psi} f=\boldsymbol{\Pi}_{\nabla \psi} \nabla f$ where

$$
\mathbf{\Pi}_{\nabla \psi} \triangleq \mathbf{I}-\nabla \psi \nabla \psi^{T}
$$

\section{Implicit Hessian}

If we compute the second derivative of $F$ we find that $\ddot{F}(0)=\nabla f(p) \cdot \ddot{\gamma}(0)+\mathbf{H}_{f}[\dot{\gamma}(0), \dot{\gamma}(0)]$. Now, we know that an arc-length parameterized geodesic curve of $\mathcal{S}$ must satisfy the harmonic maps differential equation

$$
\ddot{\gamma}+\mathbf{H}_{\psi}(\gamma)[\dot{\gamma}, \dot{\gamma}] \nabla \psi(\gamma)=0
$$

We then find that $\ddot{F}(0)=\left(\mathbf{H}_{f}(p)-\nabla f(p) \cdot \nabla \psi(p) \mathbf{H}_{\psi}(p)\right)[\dot{\gamma}, \dot{\gamma}]$. Again we have that $\dot{\gamma} \in T_{p} \mathcal{S}$, and we find the implicit Hessian of $f$ at $p$ to be

$$
\mathbf{H}_{f}^{\mathcal{S}}(p) \triangleq \boldsymbol{\Pi}_{\psi} \mathbf{h}_{f} \mathbf{\Pi}_{\psi}
$$

where

$$
\mathbf{h}_{f} \triangleq \mathbf{H}_{f}(p)-\nabla f(p) \cdot \nabla \psi(p) \mathbf{H}_{\psi}(p)
$$

We will frequently use the alternative notation $\mathbf{H}_{f}^{\psi}(p)$.

\section{Implicit Laplacian}

From the previous computation it's an easy exercise to compute the implicit Laplacian or Laplace-Beltrami of $f$ since by definition $\Delta_{\mathcal{S}} f=\operatorname{trace}\left\{\mathbf{H}_{f}^{\mathcal{S}}\right\}$.

For any pair of symmetric matrices $\mathbf{A}$ and $\mathbf{B}$ one has that $\operatorname{trace}\{\mathbf{A B A}\}=\sum_{i} \sum_{j} \sum_{k} a_{i j} a_{i k} b_{j k}$ and trace $\{\mathbf{A B}\}=\sum_{i} \sum_{j} a_{i j} b_{i j}$. Now we have that $\mathbf{\Pi}_{\psi} \mathbf{B \Pi}_{\psi}=\mathbf{B}+\nabla \psi \nabla \psi^{T} \mathbf{B} \nabla \psi \nabla \psi^{T}-\nabla \psi \nabla \psi^{T} \mathbf{B}-$ $\mathbf{B} \nabla \psi \nabla \psi^{T}$. We then obtain 


$$
\begin{aligned}
\operatorname{trace}\left\{\boldsymbol{\Pi}_{\psi} \mathbf{B} \boldsymbol{\Pi}_{\psi}\right\} & =\operatorname{trace}\{\mathbf{B}\}+\sum_{i} \sum_{j} \sum_{k} \psi_{x_{i}} \psi_{x_{j}} \psi_{x_{i}} \psi_{x_{k}} b_{j k} \\
& -2 \sum_{i} \sum_{j} \psi_{x_{i}} \psi_{x_{j}} b_{i j}
\end{aligned}
$$

Recalling that $\psi(\cdot)$ is a distance function, so that it satisfies $\|\nabla \psi\|=1$, we find

$$
\begin{aligned}
\operatorname{trace}\left\{\mathbf{\Pi}_{\psi} \mathbf{B} \mathbf{\Pi}_{\psi}\right\} & =\operatorname{trace}\{\mathbf{B}\}-\sum_{i} \sum_{j} \psi_{x_{i}} \psi_{x_{j}} b_{i j} \\
& =\operatorname{trace}\{\mathbf{B}\}-\mathbf{B}[\nabla \psi, \nabla \psi]
\end{aligned}
$$

We conclude the reasoning by taking $\mathbf{B}=\mathbf{h}_{f}$ :

$$
\begin{aligned}
\operatorname{trace}\left\{\mathbf{H}_{f}^{\mathcal{S}}\right\} & =\operatorname{trace}\left\{\mathbf{h}_{f}\right\}-\mathbf{h}_{f}[\nabla \psi, \nabla \psi] \\
& =\operatorname{trace}\left\{\mathbf{h}_{f}\right\}-\mathbf{H}_{f}[\nabla \psi, \nabla \psi]
\end{aligned}
$$

since $\mathbf{H}_{\psi}[\nabla \psi, \nabla \psi]=0$. Since trace $\left\{\mathbf{H}_{f}\right\}=\Delta f-(\nabla f \cdot \nabla \psi) \Delta \psi$, we find that

$$
\Delta_{\mathcal{S}} f=\Delta f-(\nabla f \cdot \nabla \psi) \Delta \psi-\mathbf{H}_{f}[\nabla \psi, \nabla \psi]
$$

It's interesting to observe how the expression just found for $\Delta_{\mathcal{S}} f$ coincides with the one obtained by minimizing the intrinsic Dirichlet integral, ${ }^{20}$

$$
D(f) \triangleq \frac{1}{2} \int_{\mathbb{R}^{d}}\left\|\nabla_{\mathcal{S}} f\right\|^{2} \delta(\psi) d v
$$

as is done in [3]. The authors showed that a smooth function $f$ extremizing $D(f)$ must satisfy

$$
\nabla \cdot(\nabla f-(\nabla f \cdot \nabla \psi) \nabla \psi)=0
$$

We should verify that this definition coincides with ours. This is accomplished as follows:

$$
\begin{aligned}
\nabla \cdot(\nabla f-(\nabla f \cdot \nabla \psi) \nabla \psi) & =\Delta f-(\nabla f \cdot \nabla \psi) \Delta \psi-\nabla(\nabla f \cdot \nabla \psi) \cdot \nabla \psi \\
& =\Delta f-(\nabla f \cdot \nabla \psi) \Delta \psi-\mathbf{H}_{f}[\nabla \psi, \nabla \psi]-\mathbf{H}_{\psi}[\nabla f, \nabla \psi] \\
& =\Delta f-(\nabla f \cdot \nabla \psi) \Delta \psi-\mathbf{H}_{f}[\nabla \psi, \nabla \psi] \\
& =\Delta_{\mathcal{S}} f \text { (according to our definition), }
\end{aligned}
$$

since $\mathbf{H}_{\psi}[\nabla \psi, \bullet]=0$.

\footnotetext{
${ }^{20}$ As one expects since this is the definition of harmonic functions.
} 


\section{Vector Calculus}

- Implicit Jacobian: With the ideas developed before, we easily find (differentiating $\vec{\Lambda}(t)$ ) that

$$
\mathbf{J}_{\vec{\lambda}}^{\mathcal{S}} \triangleq J_{\vec{\lambda}} \Pi_{\psi}
$$

- Implicit Divergence: Using the expression for the intrinsic Jacobian we write

$$
\nabla_{\mathcal{S}} \cdot \vec{\lambda} \triangleq \operatorname{trace}\left(\mathbf{J}_{\vec{\lambda}} \mathbf{\Pi}_{\psi}\right)
$$

and

$$
\nabla_{\mathcal{S}} \cdot \vec{\lambda} \triangleq \nabla \cdot \vec{\lambda}-\mathbf{J}_{\vec{\lambda}}[\nabla \psi, \nabla \psi]
$$

It is useful to observe that $\nabla_{\mathcal{S}} \cdot \vec{\lambda}=\nabla \cdot \vec{\lambda}$ when $\vec{\lambda}(x) \in T_{x}\{\psi=0\}$

\section{References}

[1] F. Alouges, "An energy decreasing algorithm for harmonic maps," in J.M. Coron et al., Editors, Nematics, Nato ASI Series, Kluwer Academic Publishers, Netherlands, pp. 1-13, 1991.

[2] M. Bertalmio, PhD Dissertation, University of Minnesota (www. ece.umn.edu/users/ marcelo), March 2001.

[3] M. Bertalmio, L. T. Cheng, S. Osher, and G. Sapiro, "Variational problems and partial differential equations on implicit surfaces," Journal of Computational Physics, 174:2, pp. 759-780, 2001.

[4] H. Brezis, J. M. Coron, and E. H. Lieb, "Harmonic maps with defects," Communications in Mathematical Physics 107, pp. 649-705, 1986.

[5] V. Caselles, R. Kimmel, G. Sapiro, and C. Sbert, "Minimal surfaces based object segmentation," IEEEPAMI, 19:4, pp. 394-398, April 1997.

[6] T. Chan and J. Shen, "Variational restoration of non-flat image features: Models and algorithms," UCLA CAM-TR 99-20, June 1999.

[7] Y. Chen, M.C. Hong and N. Hungerbuhler, "Heat flow of p-harmonic maps with values into spheres," Math. Z. 205, pp. 25-35, 1994.

[8] L. T. Cheng, "The level set method applied to geometrically based motion, materials science, and image processing (Ph.D. Thesis)," CAM-UCLA Report 00-20, June 2000

[9] R. Cohen, R. M. Hardt, D. Kinderlehrer, S. Y. Lin, and M. Luskin, "Minimum energy configurations for liquid crystals: Computational results," in J. L. Ericksen and D. Kinderlehrer, Editors, Theory and Applications of Liquid Crystals, pp. 99-121, IMA Volumes in Mathematics and its Applications, Springer-Verlag, New York, 1987.

[10] J. M. Corcuera and W. S. Kendall, "Riemmanian barycenters and geodesic convexity," preprint, U. of Warwick.

[11] J. M. Coron and R. Gulliver, "Minimizing p-harmonic maps into spheres," J. Reine Angew. Mathem. 401, pp. 82-100, 1989. 
[12] W. E and X. P. Wang. "Numerical Methods for the Landau-Lifshitz Equation," http://www.math.princeton.edu/ “weinan/papers/LL1.pdf

[13] M. Eck, T. DeRose, T. Duchamp, H. Hoppe, M. Lounsbery, and W. Stuetzle. "Multiresolution analysis of arbitrary meshes," Computer Graphics (SIGGRAPH '95 Proceedings), pp. 173-182, 1995.

[14] M. Eck and H. Hoppe, "Automatic reconstruction of B-spline surfaces of arbitrary topological type," Computer Graphics (SIGGRAPH), 1996.

[15] J. Eells and L. Lemarie, "A report on harmonic maps," Bull. London Math. Soc. 10:1, pp. 1-68, 1978.

[16] J. Eells and L. Lemarie, "Another report on harmonic maps," Bull. London Math. Soc. 20:5, pp. 385$524,1988$.

[17] O. Faugeras, F. Clément, R. Deriche, R. Keriven, T. Papadopoulo, J. Gomes, G. Hermosillo, P. Kornprobst, D. Lingrad, J. Roberts, T. Viéville, F. Devernay, "The inverse EEG and MEG problems: The adjoint state approach I: The continuous case," INRIA Research Report 3673, June 1999.

[18] S. F. Frisken, R. N. Perry, A. Rockwood, and T. Jones, "Adaptively sampled fields: A general representation of shape for computer graphics," Computer Graphics (SIGGRAPH), New Orleans, July 2000 .

[19] Flujos Toolbox. November 1999, http://www.iie.edu.uy/investigacion/grupos/gti/flujos/flujos.html.

[20] M. Giaquinta, G. Modica, and J. Soucek, "Variational problems for maps of bounded variation with values in $S^{1}$, " Cal. Var. 1, pp. 87-121, 1993.

[21] B. Gustafsson, H.O. Kreiss and J. Oliger, "Time Dependent Problems and Difference Methods" John Wiley \& sons inc.

[22] R. Hamilton, Harmonic maps of manifolds with boundary, Lecture Notes in Mathematics 471, Springer-Verlag, Berlin-New York, 1975.

[23] R. M. Hardt, "Singularities of harmonic maps," Bulletin of the American Mathematical Society 34:1, pp. 15-34, 1997.

[24] T. Kailath. Linear Systems, Prentice Hall, 1980.

[25] T. Kanai, H. Suzuki, and F. Kimura, "Three dimensional geometric metamorphosis based on harmonic maps,” The Visual Computer 14:4, pp.166-176, 1998.

[26] R. Kimmel and N. Sochen, "Orientation diffusion," Journal of Visual Communication and Image Representation, to appear.

[27] V. Krishnamurthy and M. Levoy, "Fitting smooth surfaces to dense polygon meshes," Computer Graphics, pp. 313-324, 1996.

[28] J. M. Lee, Riemannian Manifolds : An Introduction to Curvature, Springer Verlag, New York, 1987.

[29] F. Mémoli, Distance Maps on Implicitly Defined Manifolds, Master Thesis, Universidad de la Republica, Uruguay, May 2001. 
[30] F. Mémoli and G. Sapiro, "Fast computation of weighted distance functions and geodesics on implicit hyper-surfaces," Journal of Computational Physics, 173:2, pp. 730-764, November 2001.

[31] F. Mémoli and G. Sapiro "Harmonic brain warping," in preparation.

[32] S. Nishikawa, "On the Neumann problem for the nonlinear parabolic equation of Eells-Sampson and harmonic mappings," Math. Ann. 249, pp. 177-190, 1980.

[33] S. J. Osher and J. A. Sethian, "Fronts propagation with curvature dependent speed: Algorithms based on Hamilton-Jacobi formulations," Journal of Computational Physics 79, pp. 12-49, 1988.

[34] A. Pardo and G. Sapiro, "Vector probability diffusion," IEEE Signal Processing Letters 8, pp. 106-109, April 2001.

[35] P. Perona, “Orientation diffusion,” IEEE Trans. Image Processing 7, pp. 457-467, 1998.

[36] T. Sakai, Riemannian Geometry, AMS Translations of Mathematical Monographs, vol 149.

[37] N. Sochen, R. Kimmel, and R, Malladi, "A general framework for low level vision," IEEE Trans. Image Processing 7, pp. 310-318, 1998.

[38] M. Struwe, "On the evolution of harmonic mappings of Riemannian surfaces," Comment. Math. Helvetici 60, pp. 558-581, 1985.

[39] M. Struwe, Variational Methods, Springer Verlag, New York, 1990.

[40] B. Tang, G. Sapiro, and V. Caselles, "Diffusion of general data on non-flat manifolds via harmonic maps theory: The direction diffusion case," Int. Journal Computer Vision 36:2, pp. 149-161, February 2000 .

[41] B. Tang, G. Sapiro, and V. Caselles, "Color image enhancement via chromaticity diffusion," IEEE Trans. Image Processing 10, pp. 701-707, May 2001.

[42] J. W. Thomas, "Numerical Partial Differential Equations, Finite Difference Methods". Texts in Applied Mathematics, 22. Springer Verlag, 1995.

[43] A. W. Toga, Brain Warping, Academic Press, New York, 1998.

[44] D. Tschumperle and R. Deriche, "Regularization of orthonormal vector sets using coupled PDE's," IEEE Workshop on Variational and Level-Set Methods, Vancouver, Canada, July 2001.

[45] G. Yngve and G. Turk, "Creating smooth implicit surfaces from polygonal meshes," Technical Report GIT-GVU-99-42, Graphics, Visualization, and Usability Center. Georgia Institute of Technology, 1999.

[46] D. Zhang and M. Hebert, "Harmonic maps and their applications in surface matching," Proc. CVPR '99, Colorado, June 1999.

[47] G. Zigelman, R. Kimmel, and N. Kiryati, "Texture mapping using surface flattening via multidimensional scaling," Technion-CIS Technical Report 2000-01, 2000.

[48] L. A. Vese and S. J. Osher, "Numerical methods for p-harmonic flows and applications to image processing," CAM-UCLA Report 01-22, August 2001

[49] www.kitware.com/VTK 
[50] J. Weickert, Anisotropic Diffusion in Image Processing, ECMI Series, Teubner-Verlag, Stuttgart, Germany, 1998. 